Article

\title{
Omnidirectional Jump of a Legged Robot Based on the Behavior Mechanism of a Jumping Spider
}

\author{
Yaguang Zhu ${ }^{1,2, *}$ (D) , Long Chen ${ }^{1}$, Qiong Liu ${ }^{1}$, Rui Qin ${ }^{1}$ and Bo Jin ${ }^{2}$ \\ 1 Key Laboratory of Road Construction Technology and Equipment of MOE, Chang'an University, \\ Xi'an 710064, China; chenlong@chd.edu.cn (L.C.); liuqiong@chd.edu.cn (Q.L.); qinrui@chd.edu.cn (R.Q.) \\ 2 State Key Laboratory of Fluid Power and Mechatronic Systems, Zhejiang University, Hangzhou 310028, \\ China; bjin@zju.edu.cn \\ * Correspondence: zhuyaguang@chd.edu.cn; Tel.: +86-187-9285-2585
}

Received: 14 November 2017; Accepted: 29 December 2017; Published: 1 January 2018

Check for updates

\begin{abstract}
To find a common approach for the development of an efficient system that is able to achieve an omnidirectional jump, a jumping kinematic of a legged robot is proposed based on the behavior mechanism of a jumping spider. To satisfy the diversity of motion forms in robot jumping, a kind of 4 degrees of freedom ( $4 \mathrm{DoFs})$ mechanical leg is designed. Taking the change of joint angle as inspiration by observing the behavior of the jumping spider during the acceleration phase, a redundant constraint to solve the kinematic is obtained. A series of experiments on three types of jumping - vertical jumping, sideways jumping and forward jumping-is carried out, while the initial attitude and path planning of the robot is studied. The proposed jumping kinematic is verified on the legged robot experimental platform, and the added redundant constraint could be verified as being reasonable. The results indicate that the jumping robot could maintain stability and complete the planned task of jumping, and the proposed spider-inspired jumping strategy could easily achieve an omnidirectional jump, thus enabling the robot to avoid obstacles.
\end{abstract}

Keywords: jumping spider; jumping robot; omnidirectional jump; redundant DoF

\section{Introduction}

Compared with walking [1] and crawling robots [2], the jumping robot can walk, run, and jump [3]. Jumping locomotion has characteristics of isolated footholds, and powerful and explosive jumping force [4], which contribute to the quick and effective jumping locomotion of bio-inspired robots to stride over obstacles that are several times the size of their bodies, or cross a gully that is several times the length of their own step, and avoid danger in time. As for the jumping robot, the key point is usually planning the trajectory of their center of gravity $(\mathrm{CoG})$ to achieve the jumping process reasonably, and realize the multi-directional jumping moment in various environments. The jumping robot can realize the jumping process by controlling the take-off speed, attitude and landing stability. When the jumping robot takes off, the robot achieves certain acceleration because its foot generates enough reactive force by impacting with the ground. Then, the robot adjusts its posture in the air, and finally lands smoothly. Hence, the study of the jumping process is an important part of the jumping robot. The jumping robot realizes the physical design of the robot and the jump movements by imitating animal body structures or biological movement mechanisms. The bionics [5] include the structure bionics, the motion bionics and the control bionics [6]. Nowadays, research on the bionic robot based on a bionic structure is abundant. There are both robots that imitate mammals, such as the bionic cheetah and bionic kangaroo [7,8], and robots that imitate amphibious creatures, such as bionic 
frogs and bionic toads $[9,10]$. Additionally, there are robots that imitate arthropods, such as bionic spiders, bionic locusts, bionic cockroaches and imitation water insects [11-15]. The mammal-like robot has the characteristics of being fast at running and jumping, as well as having smooth motion and a high energy utilization rate. However, there is little research surrounding this because of its large volume and heavy weight. The amphibious robots are almost all bionic frog robots. The robot uses intermittent motion so that it may control its posture, in addition to being able to effectively control the energy accumulation and release. Therefore, this type of robot is characterized by its flexible jumping, powerful explosive force and environmental adaptability [16]. Compared with these types of robots, the arthropod robot has the advantage of fast acceleration, low energy consumption and high energy efficiency in the process of jumping because of its small size, light weight and good bounce ability [17].

The structure model of the jumping robot can be divided into a single-legged model and a multi-legged model. In terms of the single-legged jumping robot, based on the high mobility requirements of jumping, some scholars have proposed a single leg motion mode driven by hydraulics $[18,19]$. The influence posture and ground impact of the robot on its structure in the vertical jump motion are analyzed, and the overall stability evaluated, to ensure that the robot can complete the take-off task. Ge et al. [20-22] proposed a scheme based on the jumping mechanism of the kangaroo. They studied the jumping movement of the kangaroo and simplified their bodies into single-legged models to study and discuss. Then, they proposed three models: a rigid body jumping model, a compliant jump mechanism model and a rigid flexible hybrid model. The motion mechanisms of the three models were analyzed to see which one was most successful in making the robot jump smoothly. However, the single-legged model is a naturally unstable system, which cannot remain in the stationary state. Therefore, the single-legged robot has braced structures to maintain stability. Furthermore, it is necessary to adjust the initial attitude angle to achieve a smooth jump. In addition, the single-legged robots only realized the bionic jumping function, and were less involved in the overall movement mechanism. Animals in nature predominately have multiple legs. The biped robot, the quadruped robot and the six-legged robot are the main forms of multi-legged robots [23-25]. Fumitaka et al. [26] have designed a quadruped robot, which can jump even in rugged external environments and can achieve the task of crossing obstacles. Some scholars [27-29] have proposed a rigid mode of imitating the motion principle of the cricket by studying the robot's ability to jump and kick; the robot can adjust its own dynamic balance while it is jumping. Thus, compared with the single robot, the multi-legged robot has better overall stability.

Currently, most research focuses on the single direction jump, especially the vertical and forward jump. A vertical jump analysis based on the hydraulic drive has been proposed [16,30]. Surmounting ability and jumping efficiency are analyzed, and then the vertical jumping form is optimized. Thanhtam et al. [31] have proposed a new structure of quadruped robot to accomplish the task of vertical jumps or forward jumps. The joints of the robot are driven by hydraulics to meet the requirements of torque, compactness, speed and impact resistance. Hyunsoo et al. [32] proposed a quadruped jumping robot, which is based on the servo motor drive. The legs are equipped with gears, springs and other components, and the robot can complete a high jump task through two kinds of movement: spring compression and gear drive mechanism.

However, there is no in-depth discussion about the research on omnidirectional jumping. In other words, in the process of jumping, the robot jumps mainly through fixed jumping form, and it only achieves a single direction jump of height and distance. The robot must adjust its posture and jumping direction when it is trying to avoid an obstacle. The initial posture must be adjusted first if the robot is going to change the jumping direction, causing the efficiency to be greatly reduced. Hence, without changing the initial pose of the robot, multi-direction jumps become the key problem to be solved. In this paper, a bionic six-legged robot structure with the ability of omnidirectional jumping is proposed, which is based on the jumping mechanism of jumping spiders. The omnidirectional jumping form has been proposed through observing the jumping form, jumping posture and leg stretch of jumping spiders, which allows it to avoid and cross obstacles in all directions. To verify the 
rationality of the jumping form proposed in this paper, a series of experiments on a six-legged robot is carried out, and the results show that the proposed multi-direction jumping form has outstanding performance, which provided a good theoretical basis for jumping research.

\section{Bio-Inspiration and Materials}

Arthropods are the largest group of animals, including over one million species of invertebrates, and accounting for almost $84 \%$ of all species. Members of the arthropod family are various; they can be found from the abyssal sea to inland areas. Due to the differentiation between arthropod bodies, and the diversity of physical changes, which give arthropods a highly adaptive capacity, they have adapted to all sorts of surroundings, maintaining themselves even under the most rigorous conditions. After hundreds of millions of years of evolution, arthropods have become very flexible in their ability to move [33]. Arthropods can hunt prey quickly or avoid predators, and when they run into obstacles or ravines, they can quickly run and jump on the terrain to avoid obstacles. Therefore, the agility of arthropods can provide inspiration for the exploration of jumping robots [34,35]. The arthropod is composed of several different structures and functions; the body is symmetrical; its feet are evenly distributed on both sides of the body; and the legs can be coordinated with each other. This not only allows the flexibility of movement of the robot, but also the ability to jump in any direction and remain flexible in various terrains [36]. Jumping spiders are the most common of these arthropods.

Since the jumping spider has multiple joints on each leg, the legs can be stretched long enough to move around or camouflage themselves to prey. Especially due to its jumping ability, the jumping spider can quickly avoid predators and overcome obstacles. In this paper, we mainly focus on the jumping spider and study its motion structure and morphology. When the jumping spider jumps, the feet fall and the legs stretch, and the effective leg length increase rapidly, before the spider accelerates and impacts with the ground. When the jumping spider skips sideways, the front spider legs shrink and the rear legs extend in the jumping direction, and the spider has a certain attitude angle. The spider accelerates highly when its legs begin to extend. When the front legs reach the maximum effective length, the spider reaches the ground velocity across the barrier, and the spider jumps off the ground. When the jumping spider skips forward, the effective length of the front legs is constant in the direction of the jump, and the effective length of the rear legs decreases, and the spider has a certain attitude angle. The spider can achieve high acceleration when its legs extend to full extension. When the hind legs of the spider reach the maximum effective length, the spider reaches the ground velocity across the barrier, and the spider jumps off the ground.

Figure 1 is a schematic diagram of the whole body and a sketch of the spider's leg joint and leg structure. Considering the characteristics of the coxal joints of spiders, the leg structure of the robot is simplified; the blue solid line indicates the leg, and the black circle indicates the joints. The coxal joint can move freely in any direction, so that the spider can jump in any direction. The robot model is designed by observing the schematic diagram of the jumping spider and the spider leg, and the robot experiment platform is shown in Figure 2a. To simplify the design, the spider robot is designed with six legs rather than eight legs. Compared with other hexapod robots that have been studied, in this paper each leg of the robot has $4 \mathrm{DoFs}$, which can increase the flexibility of the robot during activity. The width and length of the body are $134 \mathrm{~mm}$ and $228 \mathrm{~mm}$ respectively; the length of the patella-tibia is $120 \mathrm{~mm}$; the length of the tibia-metatarsus is $120 \mathrm{~mm}$; the length of the tarsus is $160 \mathrm{~mm}$; and the maximum effective length of the robot leg is $350 \mathrm{~mm}$. The robot's six legs are symmetrically distributed on both sides of the fuselage; the angle between the legs of left foreleg (LF), right foreleg (RF), left hind leg (LH) and right hind leg (RH) (as seen in Figure 2a), and the axis direction of the robot is $60^{\circ}$, while the legs of left middle leg (LM) and right middle leg (RM) (as seen in Figure 2a) are perpendicular to the fuselage. The robot adopts the design principles of having a bionic structure and being light weight. The total weight is only $4 \mathrm{Kg}$, since the root body, shank, foot and connector of digital motors are all made from aluminum alloy. Each leg has four rotating joints including the coxal joint, complex femur-patella joint, tibia-metatarsus joint and the metatarsus-tarsus joint. The torque of each joint is 
provided by a digital motor. The rotation of the digital motion of the coxal joint $\left(\theta_{1}\right)$ enables the robot to swing back and forth, providing forward power and controlling the step size. According to the robot's mechanical structure, the minimum value of $\theta_{1}$ is $-30^{\circ}$, and the maximum value of $\theta_{1}$ is $+30^{\circ}$. The rotation of the digital motor at the femur-patella joint $\left(\theta_{2}\right)$, the tibia-metatarsus joint $\left(\theta_{3}\right)$ and the metatarsus-tarsus joint $\left(\theta_{4}\right)$ can achieve leg extension and control the height of the body. The value of $\theta_{2}$ ranges from $-90^{\circ}$ to $+75^{\circ}$, the value of $\theta_{3}$ ranges from $0^{\circ}$ to $+150^{\circ}$, and the range of $\theta_{4}$ varies with $\theta_{2}$ and $\theta_{3}$. In this paper, the robot is designed to complete tasks that require a maximum jumping height of $100 \mathrm{~mm}$ and a maximum jumping distance of $250 \mathrm{~mm}$, while following the form of omnidirectional jumping. Furthermore, the robot can walk quickly, and turn in any direction. The D-H kinematic model was established according to the John method, as shown in Figure $2 b$, and the kinematic model parameters are shown in Table 1.

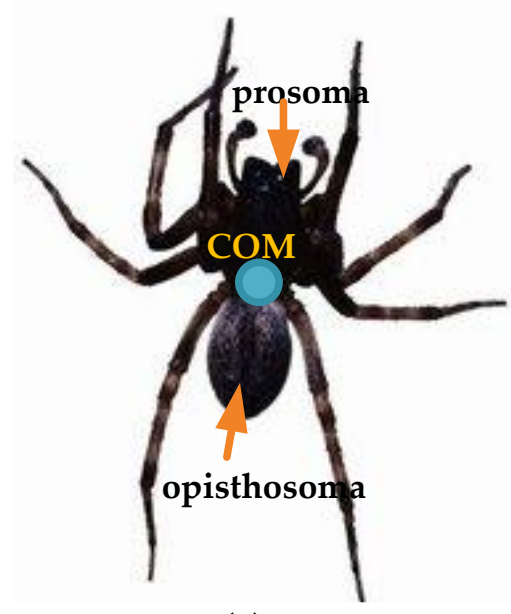

(a)

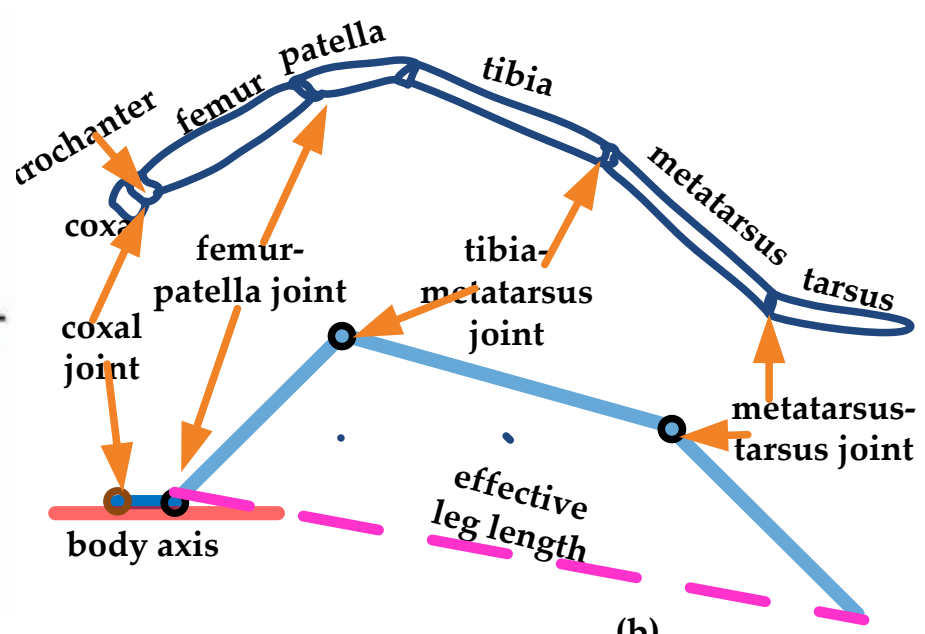

(b)

Figure 1. (a) The schematic diagram of the whole body; and (b) sketch of the spider's leg joint and leg structure.

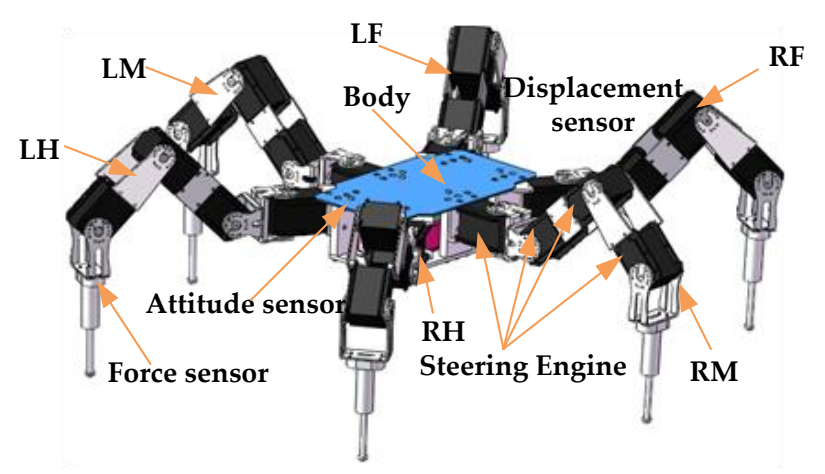

(a)

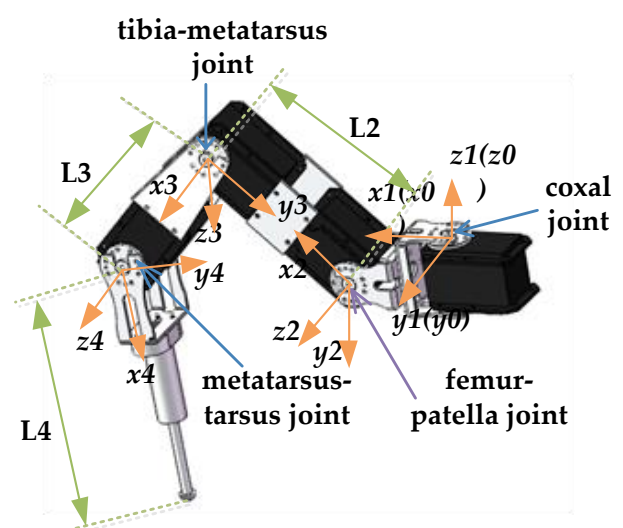

(b)

Figure 2. (a) The schematic diagram of the robot; and (b) sketch of the joint and structure.

Table 1. Kinematic model parameters.

\begin{tabular}{ccccc}
\hline Linkage (i) & $\boldsymbol{\alpha}_{\boldsymbol{j - 1}}$ & $\mathbf{d}_{\boldsymbol{j - 1}}$ & $\mathbf{a}_{\boldsymbol{j}-\mathbf{1}}$ & $\theta_{j}$ \\
\hline 1 & $0^{\circ}$ & 0 & 0 & $\theta_{1}$ \\
2 & $0^{\circ}$ & 0 & 0 & $\theta_{2}$ \\
3 & $-90^{\circ}$ & 0 & $\mathrm{~L}_{2}$ & $\theta_{3}$ \\
4 & $0^{\circ}$ & 0 & $\mathrm{~L}_{3}$ & $\theta_{3}$ \\
\hline
\end{tabular}


The architecture of the control algorithm of the robot is shown in Figure 3. During the jumping process of the robot, the whole system is composed of the time signal, path planning, foot trajectory planning, experimental prototype and the sensor signal. As the input signal of the whole system, time provides the drive for the control system. The foot trajectory planning and path planning are mainly used to plan the jumping path of the robot. When the robot jumps, the body jump trajectory is given and, correspondingly, we can get the trajectory of the robot foot. Then, the kinematic displacement of each joint is calculated by inverse kinematics. The robot can realize the motion form of the jump through the reasonable path planning of the robot. The robot is equipped with various sensors that are mounted inside the body. An attitude sensor is mounted to monitor the motion state of the robot in real time. A displacement sensor and a torque sensor are arranged on each leg joint to detect the real-time position and the torque. A force sensor is used to monitor the load on each leg. Figure 3 is the algorithm framework. We can understand clearly the control process to the jumping robot. At present, we start with the position control, which is consistent with the control mode of the robot platform we are building, and it is easy to quickly implement. The close position loop takes into account the internal torque loop, and the next step will be studied from the dynamics.

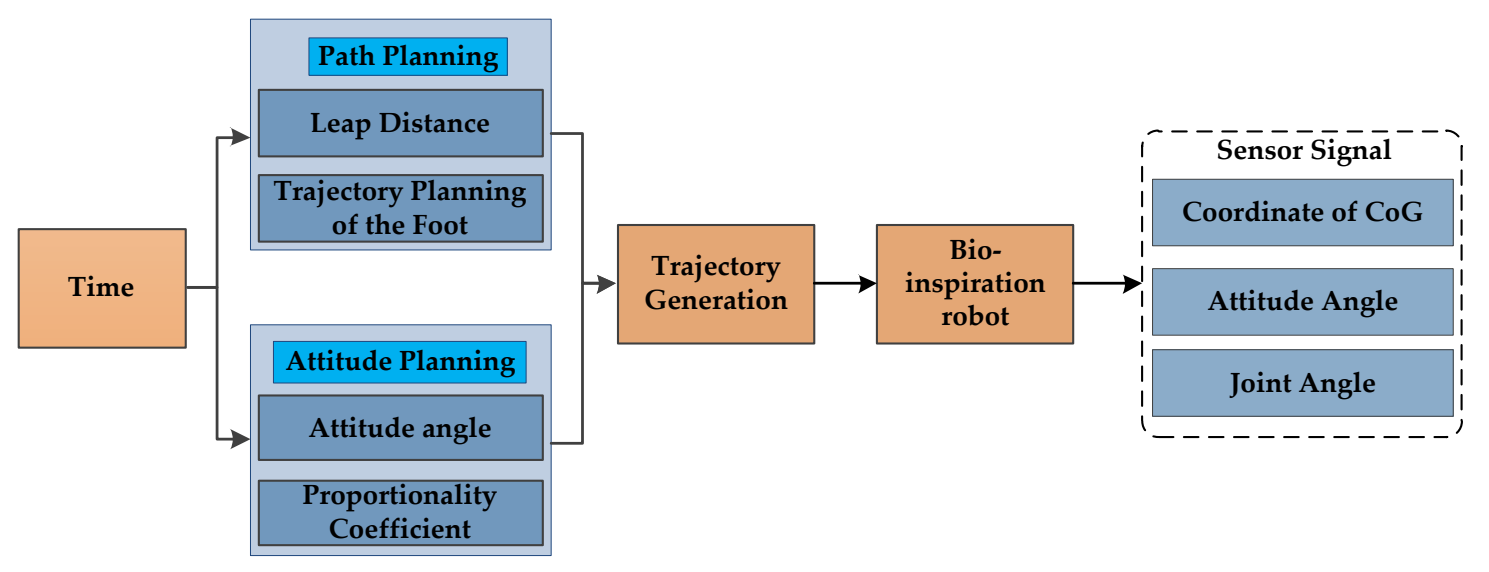

Figure 3. Algorithm framework.

\section{Methods}

\subsection{Kinematics with Redundant DoF}

\subsubsection{Forward Kinematics Analysis}

Based on the D-H model established in Section 2, the length of each link and the rotation angle of each joint are known in the base coordinate system of the robot, and the trajectory equation of the foot is derived as follows:

$$
{ }_{\text {tip }}^{0} \mathbf{P}=\left[\begin{array}{c}
0 \\
{ }_{\text {tip }} \mathrm{P}_{X} \\
{ }_{\text {tip }} \mathrm{P}_{Y} \\
{ }_{\text {tip }} \mathrm{P}_{Z}
\end{array}\right]=\left[\begin{array}{c}
\mathrm{C}_{1} \mathrm{C}_{234} \mathrm{~L}_{4}+\mathrm{C}_{1} \mathrm{C}_{23} \mathrm{~L}_{3}+\mathrm{C}_{1} \mathrm{C}_{2} \mathrm{~L}_{2} \\
\mathrm{~S}_{1} \mathrm{C}_{234} \mathrm{~L}_{4}+\mathrm{S}_{1} \mathrm{C}_{23} \mathrm{~L}_{3}+\mathrm{S}_{1} \mathrm{C}_{2} \mathrm{~L}_{2} \\
-\mathrm{S}_{234} \mathrm{~L}_{4}-\mathrm{S}_{23} \mathrm{~L}_{3}-\mathrm{S}_{2} \mathrm{~L}_{2}
\end{array}\right],
$$

is the position vector of the robot's foot relative to the reference coordinate system of the coxal joint. $\mathrm{C}_{i}=\cos \left(\theta_{i}\right), \mathrm{S}_{i}=\sin \left(\theta_{i}\right), \mathrm{C}_{i j}=\cos \left(\theta_{i}+\theta_{j}\right), \mathrm{S}_{i j}=\sin \left(\theta_{i}+\theta_{j}\right), \mathrm{C}_{i j k}=\cos \left(\theta_{i}+\theta_{j}+\theta_{k}\right)$.

\subsubsection{Inverse Kinematics Analysis}

According to the position vector of the robot foot relative to the reference coordinate system of the coxal joint, the four joint angles of the leg can be obtained. However, the four joint angles can 
be driven directly only through being given the position vector of the foot. Therefore, a constraint is added. Firstly, the rotation angle of the coxal joint $\left(\theta_{1}\right)$ is solved by the algebraic method

$$
\theta_{1}=\operatorname{atan}\left(\frac{{ }_{\text {tip }} \mathrm{P}_{\mathrm{Y}}}{0} \mathrm{PX}_{\mathrm{X}}\right) .
$$

The rotation angles of the femur-patella joint $\left(\theta_{2}\right)$, the tibia-metatarsus joint $\left(\theta_{3}\right)$ and the metatarsus-tarsus joint $\left(\theta_{4}\right)$ are solved by the geometric method. The base coordinate system $\{O\}$ is assumed to attach to the coordinate system of the coxal joint. The mechanical leg is projected in the $\mathrm{X}-\mathrm{Z}$ plane coordinate system, and the simplified model of the linkage is shown in Figure 4.

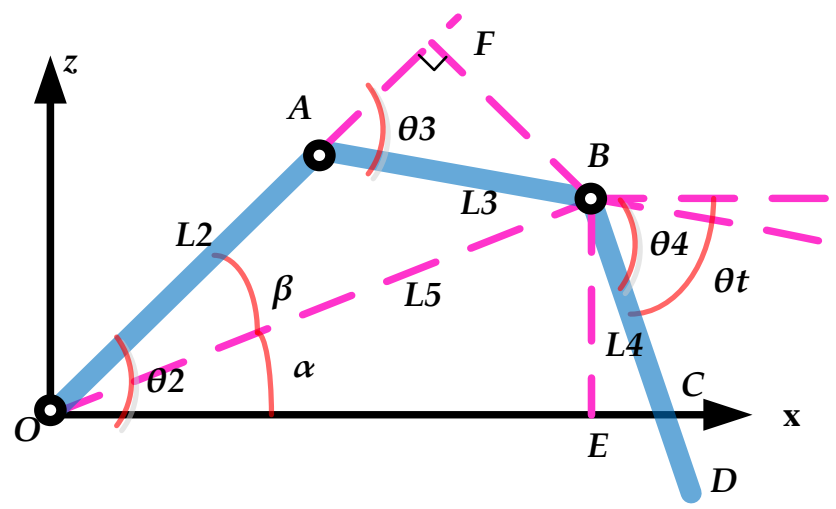

Figure 4. The simplified model of the linkage. O represents the femoral-patella joint, A represents the tibia-metatarsus joint, B represents the metatarsus-tarsus joint, and D represents the foot tip of the robot.

In Figure 4, O represents the femoral-patella joint, A represents the tibia-metatarsus joint and B represents the metatarsus-tarsus joint. We have aligned the coxal joint and the femoral-patella joint at $\mathrm{O}$ for convenience in calculating the angles. The angle between the connecting rod $\mathrm{BD}$ and the $\mathrm{X}$ axis or the ground is $\theta_{t} ; \theta_{t}$ is the attitude angle of the foot. The geometric relation between the links of the linkage mechanism can be obtained by $\theta_{t}$ and $\theta_{2}, \theta_{3}$ and $\theta_{4}$, which satisfy the constraint relation in Equation (3)

$$
\theta_{t}=\theta_{2}+\theta_{3}+\theta_{4}
$$

The plane coordinate of $\mathrm{D}$ relative to $\mathrm{O}$ is $\left(\mathrm{P}_{X}, \mathrm{P}_{Z}\right)$. According to the projection principle, the relation between $\mathrm{P}_{X}, \mathrm{P}_{Z}$ and ${ }_{\text {tip }}^{0} \mathrm{P}_{X},{ }_{\text {tip }}^{0} \mathrm{P}_{Z}$ is

$$
\left\{\begin{array}{l}
\mathrm{P}_{\mathrm{X}}={ }_{\text {tip }}^{0} \mathrm{P}_{\mathrm{X}} / \cos \theta_{1} \\
\mathrm{P}_{\mathrm{Z}}={ }_{\text {tip }}^{0} \mathrm{P}_{\mathrm{Z}}
\end{array} .\right.
$$

In the $\triangle \mathrm{OBE}$, according to cosine theorem, length $L_{5}$ between $\mathrm{O}$ and $\mathrm{B}$ is

$$
\mathrm{L}_{5}=\sqrt{\left(\mathrm{P}_{\mathrm{X}}-\mathrm{L}_{4} \cos \left(\theta_{\mathrm{t}}\right)\right)^{2}+\left(\mathrm{P}_{\mathrm{Z}}+\mathrm{L}_{4} \sin \left(\theta_{\mathrm{t}}\right)\right)^{2}} .
$$

In the $\triangle \mathrm{OAB}$, the $\theta_{3}$ holds

$$
\theta_{3}=\arccos \left(\frac{\mathrm{L}_{5}{ }^{2}-\mathrm{L}_{2}{ }^{2}-\mathrm{L}_{3}{ }^{2}}{2 \mathrm{~L}_{2} \mathrm{~L}_{3}}\right) .
$$


In the $\triangle \mathrm{OBE}$

$$
\tan \alpha=\frac{\left(\mathrm{L}_{4} \sin \left(\theta_{t}\right)+\mathrm{P}_{\mathrm{Z}}\right)}{\mathrm{P}_{\mathrm{X}}-\mathrm{L}_{4} \cos \left(\theta_{\mathrm{t}}\right)}, \alpha=-\arctan \left(\frac{\mathrm{P}_{\mathrm{Z}}+\mathrm{L}_{4} \sin \left(\theta_{t}\right)}{\mathrm{P}_{\mathrm{X}}-\mathrm{L}_{4} \cos \left(\theta_{t}\right)}\right) .
$$

In $\triangle \mathrm{OBF}$ and $\triangle \mathrm{ABF}$, they meet the following formula

$$
\beta=-\arcsin \left(\frac{L_{3} \sin \left(\theta_{3}\right)}{L_{5}}\right) .
$$

Thus, we can obtain

$$
\theta_{2}=\alpha+\beta=-\arctan \left(\frac{P_{Z}+L_{4} \sin \left(\theta_{t}\right)}{P_{X}-L_{4} \cos \left(\theta_{t}\right)}\right)-\arcsin \left(\frac{L_{3} \sin \left(\theta_{3}\right)}{L_{5}}\right) .
$$

From the constraints relation above, $\theta_{3}$ solutions can be

$$
\theta_{4}=\theta_{t}-\theta_{2}-\theta_{3}
$$

Hence, the inverse kinematics is

$$
\left\{\begin{array}{l}
\theta_{1}={ }_{\text {tip }}^{0} \mathrm{P}_{\mathrm{T}} \\
\operatorname{tip}_{2} \mathrm{P}_{2} \\
\theta_{2}=-\arctan \left(\frac{\mathrm{P}_{\mathrm{Z}}+\mathrm{L}_{4} \sin \left(\theta_{\mathrm{t}}\right)}{\mathrm{P}_{\mathrm{X}}-\mathrm{L}_{4} \cos \left(\theta_{\mathrm{t}}\right)}\right)-\arcsin \left(\frac{\mathrm{L}_{3} \sin \left(\theta_{3}\right)}{\mathrm{L}_{5}}\right) \\
\theta_{3}=\arcsin \left(\frac{\mathrm{L}_{5}{ }^{2}-\mathrm{L}_{2}{ }^{2}-\mathrm{L}_{3}{ }^{2}}{2 \mathrm{~L}_{2} \mathrm{~L}_{3}}\right) \\
\theta_{4}=\theta_{\mathrm{t}}-\theta_{2}-\theta_{3}
\end{array} .\right.
$$

In this paper, we proposed a study based on bionic kinematics with redundant freedom. Figure 5 provides a sketch of the leg flexion and the extension of the spider. The attitude angle of the foot $\theta_{t}$ is required to solve the leg joint angles. Thus, the attitude angle of the foot can be obtained by using the relationships between the changes of joint angles and the foot posture during jumping. In the jumping process of the spider, the rapid extension of the leg makes the effective leg length increase rapidly to complete the fast jumping. Simultaneously, the joint angle of the spider leg changes regularly with the effective length, and the attitude angle of the foot vary regularly with the joint angle. Figure 5 shows a sketch of the leg flexion and the extension of the spider.

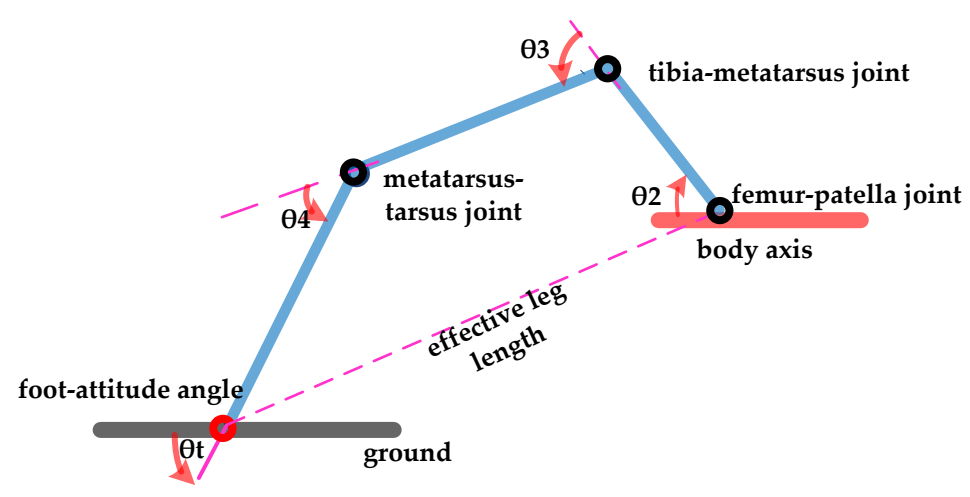

Figure 5. Schematic of the leg at defined instances. Black circles indicate the joint and the red circle indicates the foot. The arrows indicate the movements of the joints. The red line represents the axis of the spider's body, the blue line represents the leg of spider, and black line represents the ground.

Figure 6 shows the angle curve for the duration of the acceleration phase. During the jump process, the spider completes the jump in a fixed pattern. The effective leg length, the body attitude angle, the joint angle and the foot stance angle determine the maximum values of the take-off form, direction, 
height and distance. The leg extends in the jumping direction during its contact phase, which allows the spider to quickly accumulate speed, while the posture of the spider's body changes depending on the jumping direction, and the attitude angle varies with different jumping forms. When a spider jumps upward, the effective stretch length of each leg is the same, and the body posture hardly changes. When the spider jumps sideways, the body has a certain initial roll angle. At the same time, the length of the hind leg is rapidly stretched along the back of the jumping direction and the effective length of the leg increases rapidly. Then, the forelegs stretch in the jumping direction to achieve the sideways jump. In the process of the sideways jump, the length of the hind legs is longer than that of the forelegs of the spider in the jumping direction and, as a result, the rolling angle of the spider is larger. When the spider jumps forward, it has a certain initial pitch angle in the jump direction. At the same time, the hind legs extend rapidly at first; the effective length of the legs increases rapidly, and then the forelegs rapidly extend to achieve the forward jump. In the whole process of the forward jump, the pitch angle of the body posture becomes bigger. In this paper, we study the kinematic of the jumping spider [7] by observing and studying the relationships between the changes of joint angles and effective leg length during the jumping process. Using the experimental data and the curve of the jumping process of the spider, some groups of jumping motion curves are analyzed, and then several sets of the joint angle curve are fitted out in the interval angle of each joint. According to the D-H kinematic model in the last section and the definition of joint angles, the mathematical relationship between the joint angles of the jumping spider and the joint angles of the robot can be obtained. A suitable angle curve and the attitude angle of the foot are used for the spider-inspired robot. Figure 6 shows the angle curve of the femur-patella joint, femur-patella joint, the metatarsus-tarsus joint and the attitude angle of the foot for the robot during the acceleration phase. Similarly, in the robot's acceleration phase during take-off, the angles of each joint change with the extension of the leg, including the angle of the femoral-patella joint which increases gradually by approximately $90^{\circ}$. The tibia-metatarsal angle decreases gradually by approximately $55^{\circ}$; the metatarsus-tarsus angle decreases gradually and the angle varies by $35^{\circ}$; and the attitude angle of the feet is kept within $70-80^{\circ}$. Through observing the black solid line, the constraint is obtained

$$
\theta_{t}=-0.02 \mathrm{t}+73^{\circ}
$$

Hence, we propose the method based on bionic kinematics with redundant freedom as it offers an excellent approach to solve the inverse kinematics for a bionic structure with redundant freedom. 


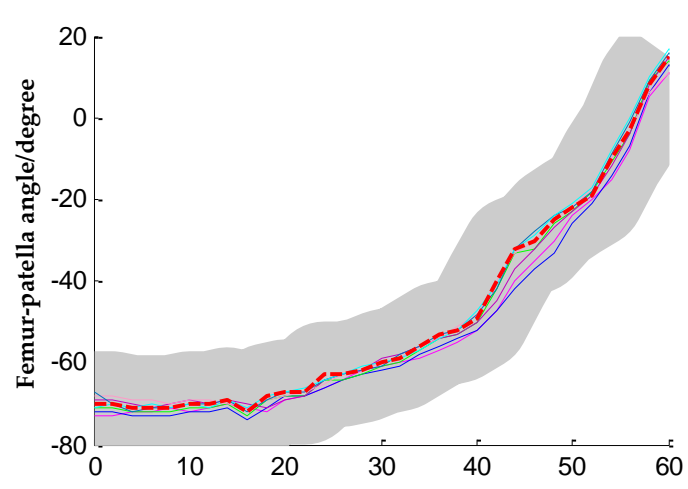

(a)

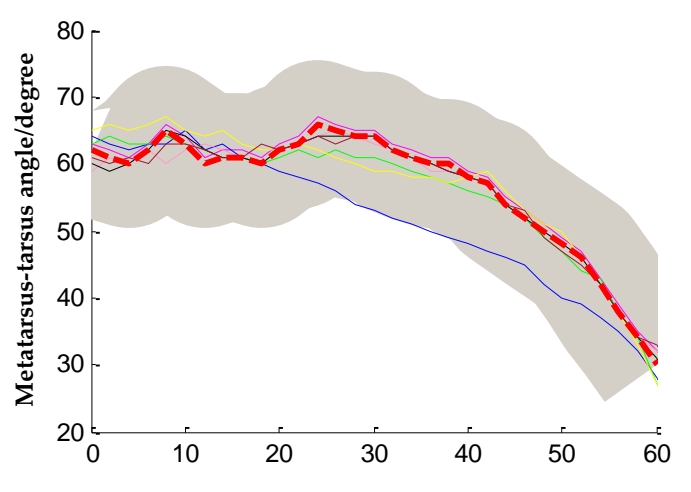

(c)

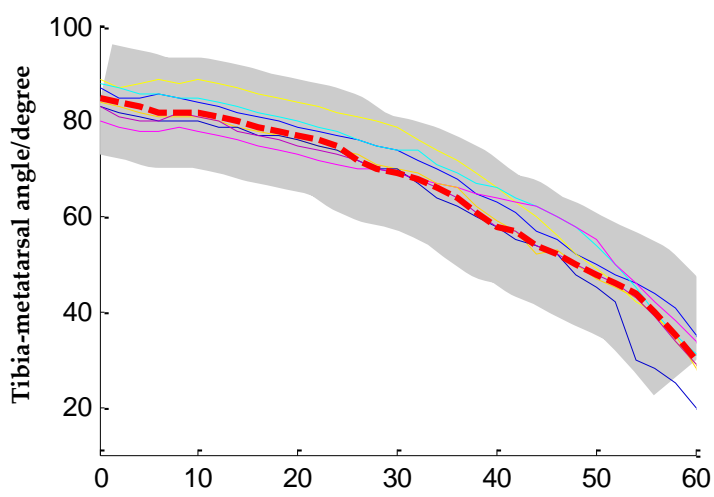

(b)

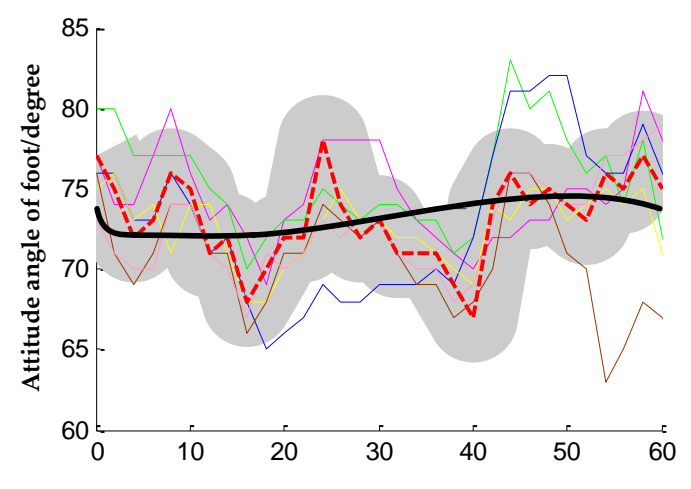

(d)

Time/ms

Figure 6. Angle curve for the duration of the acceleration phase: (a) femur-patella angle; (b) femur-patella angle; (c) metatarsus-tarsus angle; and (d) attitude angle of the foot. The red dashed line indicates the optimal curve, and the shadow area indicates the range of the angle of the joint indicated by the Quartile Range (IQR) method. The black solid line indicates the optimal curve of the attitude angle of the foot. The other solid line indicates the angle curve for the duration of the acceleration phase.

\subsection{Locomotion Planning}

\subsubsection{Path Planning}

There are three phases in the whole jumping process: take-off, flying and touchdown. In this paper, it is mainly the take-off phase of the robot that is studied. The take-off phase refers to the whole movement process of the robot, from a stationary state to jumping away from the ground. In other words, from the moment the robot foot has been subjected to the ground force $F_{g}$ in the take-off phase until the moment the robot's feet leave the ground. The take-off process of jumping consists of flexing the leg to store energy and extending to release energy. In the first phase, the legs flex and the CoG drops. Then, the initial attitude angle is adjusted with stored energy to take off. In the phase of releasing energy, the robot legs extend, the body mass center rises, and the robot adjusts to reach a proper take-off stance. Then, the legs continue to extend, hit the ground violently and cause impact force, which makes the robot accelerate and realize the jumping movement. Therefore, the trajectory of the mass center decreases slowly and then rises rapidly. When the robot jumps, the speed of the CoG is an important index of the take-off performance of a robot, as it determines the jumping height and the jumping distance when the robot jumps off the ground. Suppose that the moment the robot takes off from the ground is $t_{f}$, the velocity of the CoG is $[\dot{X}(t) \dot{Y}(t) \dot{Z}(t)]^{T}$, and the acceleration is $[\ddot{X}(t) \ddot{Y}(t) \ddot{Z}(t)]^{T}$. The foot of the robot generates the impact force via continuous contact with the ground so that the robot will accumulate acceleration and speed and finally realize the jumping task. 
In this paper, three different types of jumping—vertical jumping, side jumping and forward jumping-are discussed. The position vector of the robot foot relative to the base coordinate is $[\mathrm{XYZ} Z]^{\mathrm{T}}$. When the robot jumps vertically, the whole body has an upward acceleration and velocity relative to the ground. The robot has acceleration and velocity in the $\mathrm{Z}$ direction. When the robot jumps sideways, the robot has acceleration and velocity in the $X$ and $Z$ direction. When the robot jumps forward, the robot has acceleration and velocity only in the $\mathrm{Y}$ and $\mathrm{Z}$ direction. Thus, the constraint conditions of the motion of the CoG in these jumping forms of the robot must be discussed. At first, the reacting force of the ground to the foot of the robot gradually decreases to zero when the foot of the robot leaves the ground. At this instant, the contact force of the foot fulfills the condition

$$
\mathrm{F}_{\mathrm{gx}}=\mathrm{F}_{\mathrm{gy}}=\mathrm{F}_{\mathrm{gz}}=0 .
$$

The acceleration of CoG fulfills the following constraints:

$$
\left.\begin{array}{c}
\ddot{\mathrm{X}}_{\mathrm{f}}=0 \\
\ddot{\mathrm{Y}}_{\mathrm{f}}=0 \\
\ddot{\mathrm{Z}}_{\mathrm{f}}=-\mathrm{g}
\end{array}\right\} \text {. }
$$

At the take-off moment $t_{f}$, the robot accumulates sufficient velocity to achieve three types of jumps and the robot leaves the ground. The velocity constraints are as shown in Table 2. Here, $V_{x u f}$, $\mathrm{V}_{\text {yuf }}$, and $\mathrm{V}_{\text {zuf }}$ denote the velocity of the vertical jump. $\mathrm{V}_{\mathrm{xsf}}, \mathrm{V}_{\mathrm{ysf}}$, and $\mathrm{V}_{\mathrm{zsf}}$ denote the velocity of the sideways jump. $\mathrm{V}_{\mathrm{xff}}, \mathrm{V}_{\mathrm{yff}}$, and $\mathrm{V}_{\mathrm{zff}}$ denote the velocity of the forward jump. Therefore,

$$
\left.\left.\left.\begin{array}{r}
\mathrm{V}_{\text {xuf }}=\dot{\mathrm{X}}_{\mathrm{f}} \\
\mathrm{V}_{\text {yuf }}=\dot{\mathrm{Y}}_{\mathrm{f}} \\
\mathrm{V}_{\text {zuf }}=\dot{\mathrm{Z}}_{\mathrm{f}}
\end{array}\right\}, \begin{array}{r}
\mathrm{V}_{\text {xuf }}=\dot{\mathrm{X}}_{\mathrm{f}} \\
\mathrm{V}_{\text {yuf }}=\dot{\mathrm{Y}}_{\mathrm{f}} \\
\mathrm{V}_{\mathrm{zuf}}=\dot{\mathrm{Z}}_{\mathrm{f}}
\end{array}\right\}, \begin{array}{r}
\mathrm{V}_{\text {xuf }}=\dot{\mathrm{X}}_{\mathrm{f}} \\
\mathrm{V}_{\text {yuf }}=\dot{\mathrm{Y}}_{\mathrm{f}} \\
\mathrm{V}_{\text {zuf }}=\dot{\mathrm{Z}}_{\mathrm{f}}
\end{array}\right\}
$$

Table 2. Velocity constraints.

\begin{tabular}{ccc}
\hline Upward Jumping & Sideway Jumping & Forward Jumping \\
\hline $\mathrm{V}_{\mathrm{xuf}}=0$ & $\mathrm{~V}_{\mathrm{xsf}}>0$ & $\mathrm{~V}_{\mathrm{xff}}=0$ \\
$\mathrm{~V}_{\mathrm{yuf}}=0$ & $\mathrm{~V}_{\mathrm{xsf}}=0$ & $\mathrm{~V}_{\mathrm{xff}}>0$ \\
$\mathrm{~V}_{\text {zuf }}>0$ & $\mathrm{~V}_{\mathrm{xsf}}>0$ & $\mathrm{~V}_{\mathrm{xff}}=0$ \\
\hline
\end{tabular}

During the whole process of the take-off $\left(0 \leq t \leq t_{\mathrm{f}}\right)$, it is necessary to ensure that the robot does not leave the ground in advance and that it gains sufficient take-off speed. $a_{x u f}, a_{y u f}$, and $a_{\text {zuf }}$ are used to indicate the acceleration of the robot when the robot jumps vertically. $a_{x s f}, a_{y s f}$, and $a_{z s f}$ are used to indicate the acceleration of the robot when the robot jumps sideways. $a_{x f f}, a_{y f f}$, and $a_{z f f}$ are used to indicate the acceleration of the robot when the robot jumps forward. The acceleration constraints are as shown in Table 3. Therefore,

$$
\left.\left.\left.\begin{array}{l}
a_{\text {xuf }}=\ddot{X}_{f} \\
a_{\text {yuf }}=\ddot{Y}_{f} \\
a_{\text {zuf }}=\ddot{Z}_{f}
\end{array}\right\}, \begin{array}{l}
a_{\text {xsf }}=\ddot{X}_{f} \\
a_{\text {ysf }}=\ddot{Y}_{f} \\
a_{\text {zsf }}=\ddot{Z}_{f}
\end{array}\right\}, \begin{array}{l}
a_{\text {xff }}=\ddot{X}_{f} \\
a_{\text {yff }}=\ddot{Y}_{f} \\
a_{\text {zff }}=\ddot{Z}_{f}
\end{array}\right\} .
$$


Table 3. Acceleration constraints.

\begin{tabular}{ccc}
\hline Upward Jumping & Sideway Jumping & Forward Jumping \\
\hline $\mathrm{a}_{\mathrm{xuf}}=0$ & $\mathrm{a}_{\mathrm{xsf}}>0$ & $\mathrm{a}_{\mathrm{xff}}=0$ \\
$\mathrm{a}_{\mathrm{yuf}}=0$ & $\mathrm{a}_{\mathrm{xff}}=0$ & $\mathrm{a}_{\mathrm{xff}}>0$ \\
$\mathrm{a}_{\mathrm{zuf}}>0$ & $\mathrm{a}_{\mathrm{xsf}}>0$ & $\mathrm{a}_{\mathrm{xff}}=0$ \\
\hline
\end{tabular}

When the robot is ready to take off, the motion of the foot relative to the base coordinate $\{\mathrm{O}\}$ should fulfill the following boundary conditions

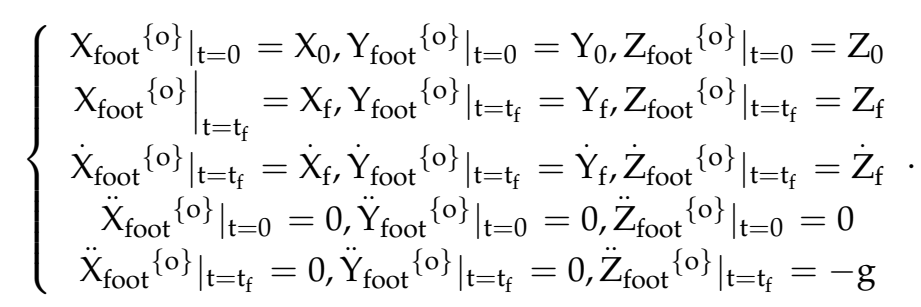

where $\left[\mathrm{X}_{0} \mathrm{Y}_{0} \mathrm{Z}_{0}\right]^{\mathrm{T}}$ is the position vector of the foot relative to the base coordinate $\{\mathrm{O}\}$ at the initial stage of jumping, and $\left[X_{f} Y_{f} Z_{f}\right]^{T}$ is the position vector of the foot relative to the base coordinate $\{O\}$ when the robot leaves the ground.

Then, the robot has the following relation when it is in the flying phase:

$$
\left\{\begin{array}{l}
\mathrm{L}_{\mathrm{x}}=2 \dot{\mathrm{X}}_{\mathrm{f}} \dot{\mathrm{Z}}_{\mathrm{f}} / \mathrm{g} \\
\mathrm{L}_{\mathrm{y}}=2 \dot{\mathrm{Y}}_{\mathrm{f}} \dot{\mathrm{Z}}_{\mathrm{f}} / \mathrm{g} \\
\mathrm{H}_{\mathrm{V}}=\dot{\mathrm{Z}}_{\mathrm{f}}^{2} / 2 \mathrm{~g}
\end{array}\right.
$$

where $\mathrm{H}_{\mathrm{v}}$ is the maximum jumping height of the robot, $\mathrm{L}_{\mathrm{y}}$ is the maximum distance along the longitudinal body axis, and $\mathrm{L}_{\mathrm{x}}$ is the maximum distance along the body lateral axis. During the take-off process, the rational trajectory planning of the foot enables the robot to complete the jump task successfully. The acceleration of the robot while jumping is planned. To make sure that there is no impact between the foot and the ground when the robot makes contact and lifts off, the contact force must be smooth. Here, the quadric curve is used to plan the acceleration of the robot. Then, the acceleration curve equations are

$$
\left\{\begin{array}{l}
a_{x}=b_{0 x}+b_{1 x} t_{1}+b_{2 x} t_{1}{ }^{2} \\
a_{y}=b_{0 x}+b_{1 x} t_{1}+b_{2 x} t_{1}{ }^{2} \\
a_{z}=b_{0 z}+b_{1 z} t_{1}+b_{2 z} t_{1}{ }^{2}
\end{array} .\right.
$$

where the symbols $t_{1}, t_{2}$, and $t_{3}$, respectively, indicate the initial time $t_{0}$, the ground departure time $\mathrm{t}_{\mathrm{f}}$ and the intermediate time $\mathrm{t}_{\mathrm{m}}$ of the body. The symbols $\left(\mathrm{a}_{\mathrm{x} 1}, \mathrm{a}_{\mathrm{y} 1}, \mathrm{a}_{\mathrm{z} 1}\right),\left(\mathrm{a}_{\mathrm{x} 2}, \mathrm{a}_{\mathrm{y} 2}, \mathrm{a}_{\mathrm{z} 2}\right)$, and $\left(\mathrm{a}_{\mathrm{x} 3}, \mathrm{a}_{\mathrm{y} 3}, \mathrm{a}_{\mathrm{z} 3}\right)$, respectively, indicate the acceleration vectors corresponding to the three moments. The coefficients of each component are

$$
\mathrm{B}=[\mathrm{T}]^{-1} \cdot[\mathrm{A}] .
$$

where $[\mathrm{T}]$ is time matrix, $[\mathrm{A}]$ is coordinate matrix. According to the planning of the acceleration, the instantaneous velocity when the robot foot hits the ground and the trajectory of the foot tip can be obtained as

$$
\left\{\begin{array}{l}
\mathrm{X}(\mathrm{t})=\iint \mathrm{a}_{\mathrm{x}} \mathrm{dt} \\
\mathrm{Y}(\mathrm{t})=\iint \mathrm{a}_{\mathrm{y}} \mathrm{dt} \\
\mathrm{Z}(\mathrm{t})=\iint \mathrm{a}_{\mathrm{z}} \mathrm{dt}
\end{array},\left\{\begin{array}{r}
\mathrm{v}(\mathrm{t})=\int \mathrm{a}_{\mathrm{x}} \mathrm{dt} \\
\mathrm{v}(\mathrm{t})=\int \mathrm{a}_{\mathrm{y}} \mathrm{dt} \\
\mathrm{v}(\mathrm{t})=\int \mathrm{a}_{\mathrm{z}} \mathrm{dt}
\end{array} .\right.\right.
$$

According to the position vector of the robot foot relative to the base coordinate $[\mathrm{X} \mathrm{Y} \mathrm{Z}]^{\mathrm{T}}$, we can obtain all joint angles of the robot by inverse kinematics. The acceleration of the robot becomes zero 
at the exact moment of the foot's take-off, and the velocity of the robot reaches its maximum at the same time. During the flying phase of the robot, the maximum jumping height of the robot is $100 \mathrm{~mm}$, the maximum distance along the longitudinal body axis is $250 \mathrm{~mm}$, and the maximum distance along the body lateral axis is $250 \mathrm{~mm}$.

\subsubsection{Attitude Planning}

In the jump process of the robot, the reasonable initial attitude angle of the robot can affect the jumping height and distance. The initial jumping posture mainly includes the pitch angle, yaw angle and roll angle of the robot when taking off. By controlling the pitch angle of the robot, the maximum height and maximum distance of the robot can be reached when the robot jumps forward. The roll angle affects the sideways jump, while the yaw angle can adjust the jumping direction of the robot, allowing the robot to jump towards the target. During vertical jumps, the yaw angle and pitch angle of the robot are $0^{\circ}$. The initial joint position and angles of each leg of the robot are the same, correspondingly. The robot can jump vertically by reacting to the ground. Before the robot begins jumping sideways, the body leans to the left due to a slight flexion of the left legs, and the robot has a certain roll angle of $\theta_{r}$ in the jumping direction. Meanwhile, the yaw angle and pitch angle are zero. A simple model of the robot's posture during a sideways jump is shown in Figure 7a. When the robot jumps to the right, the effective length of the three left legs of the robot is larger than that of the right legs in the initial state to satisfy a certain proportion. Therefore, we can obtain

$$
\mathrm{Z}_{1}=\mathrm{K}_{1} \mathrm{Z}_{\mathrm{r}}
$$

where $Z_{1}$ is the $Z$ coordination of the three left legs opposite to the base coordinate $\{O\}$. $Z_{r}$ is for the right legs. $K_{1}$ is a constant, and it is determined by the initial attitude of the robot.

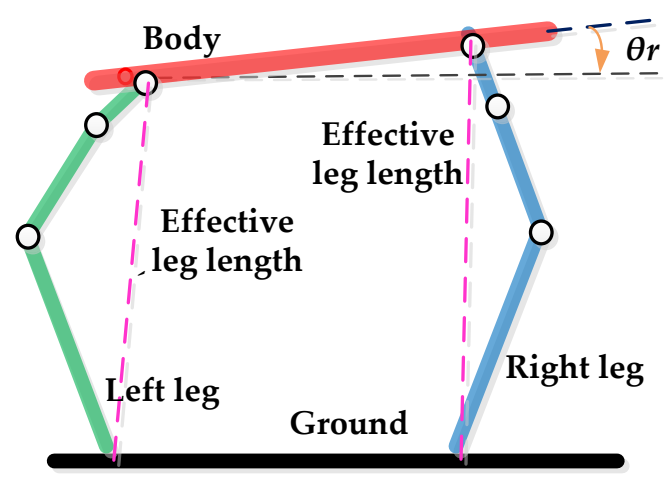

(a)

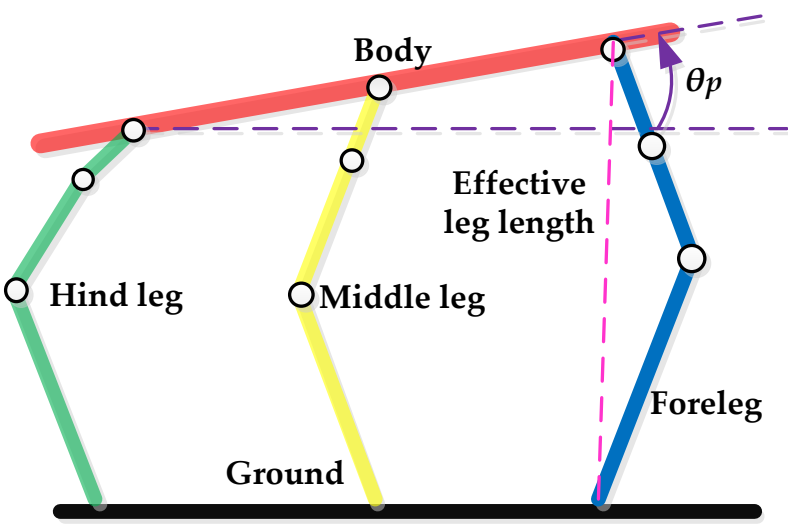

(b)

Figure 7. Simplified model of the robot posture. (a) Sideways jump: The red solid line indicates the transverse axis of the robot body, the green solid line indicates the left leg of the robot body, the blue solid line indicates the right leg, the purple dashed line indicates the effective length, and the black circles indicate the joints. (b) Jump forward: Green solid lines indicate the hind legs of the robot body, the blue solid line indicates the foreleg, and the yellow solid line indicates the middle leg.

When the robot jumps forward, the forelegs extend and the hind legs flex during their contact phase, and the middle legs remain in slight extension. Therefore, the robot has a certain pitch angle in the jump direction; the pitch angle is $\theta_{\mathrm{p}}$. The roll angle and raw angle are $0^{\circ}$. The simple model of the robot posture of the forward jump is shown in Figure $7 \mathrm{~b}$. At the beginning of the jump, the effective length of the forelegs of the robot is larger than that of the middle legs in the initial state; meanwhile, 
the effective length of the middle legs of the robot is larger than that of the hind legs in the initial state, and they satisfy two proportions:

$$
\left\{\begin{array}{l}
Z_{f}=K_{2} Z_{m} \\
Z_{m}=K_{2} Z_{h}
\end{array},\right.
$$

where $Z_{h}$ is the $Z$ coordination of the hind legs opposite to the base coordinate $\{O\} ; Z_{f}$ is the forelegs; and $Z_{m}$ is the middle legs. $K_{2}$ is a constant and it is determined by the initial attitude of the robot.

\section{Results}

To further verify the algorithm of omnidirectional jumping, a series of experiments is conducted using a hexapod robot platform. There are three groups of experiments: the vertical jump, sideways jump, and forward jump. In these experiments, the joint angles as input of the simulation system, and the CoG and the attitude angle as output of the simulation system are used to verify the design requirement and stability of the robot. To avoid the slip phenomenon in the jumping process, and to achieve a certain friction between the ground and the robot foot, we use a wooden floor as the ground. We assume that there is no air resistance and no slipping phenomenon when the robot jumps, since the beginning of the jump is our main concern and the structure is slim with little air resistance. Friction is supposed as a load torque. Robot links are in rigid connections. In this paper, the simulation results are obtained under Matlab-Adams, relevant parameters are set in Adams, and the parameters include static coefficient, dynamic coefficient and stiffness. Experiment parameters are shown in Table 4.

Table 4. Experiment parameters.

\begin{tabular}{ccc}
\hline Parameters & Symbol & Value \\
\hline Jumping height & $\mathrm{H}_{\mathrm{v}}$ & $100 \mathrm{~mm}$ \\
Jumping distance & $\mathrm{L}_{\mathrm{x}}\left(\mathrm{L}_{\mathrm{y}}\right)$ & $250 \mathrm{~mm}$ \\
Static coefficient & $\mathrm{u}_{\mathrm{S}}$ & 0.35 \\
Dynamic coefficient & $\mathrm{u}_{\mathrm{D}}$ & 0.2 \\
Stiffness & $\mathrm{T}$ & $10000 \mathrm{~N} / \mathrm{m}$ \\
Take-off moment & $\mathrm{t}_{\mathrm{f}}$ & $1 \mathrm{~s}$ \\
\hline
\end{tabular}

\subsection{Vertical Jump}

In this section, we obtain data on the robot jumping vertically; these data include the joint angle, attitude angle, joint velocity, foot contact force, take-off velocity, trajectory of CoG, jumping height and jumping distance. These data are based on the simulation result under Matlab-Adams, and we can prove the reliability and rationality of vertical jump by analyzing it.

Figure $8 \mathrm{a}, \mathrm{b}$ shows the simulation platform of the virtual jump prototype. At the beginning of the vertical jump, the body slowly moves downward due to a slight flexion of the robot leg, and the robot begins to store energy. During the acceleration phase of the vertical jump, the effective leg length extends quickly, and the robot starts to take off. Figure $8 \mathrm{c}$ shows a sketch of the robot jumping vertically. The initial height of the CoG of the robot is $180 \mathrm{~mm}$ and the jumping height is $100 \mathrm{~mm}$. In the vertical jumping experiment, we measure the contact force of the foot and the joint force; we observe the change of joint forces to verify whether the motors meet the requirement.

During the vertical jumping, each joint of the six legs has the same rotation angle. Here, we can see the set of angle curves of the robot leg in Figure 8c. Coxal joint angles of all legs always remain the same during the vertical jump: they are 0 rad. Before the acceleration phase begins, the femur-patella joint angle, tibia-metatarsus joint angle and metatarsus-tarsus joint angle begin to slowly change, and the robot body slows down and adjusts the attitude. Then, the foot moves down quickly, and the robot starts to accelerate by extending its legs at $0.8 \mathrm{~s}$. At $1.0 \mathrm{~s}$, the height of the CoG is $300 \mathrm{~mm}$, and the robot leaves the ground and takes off. At $1.2 \mathrm{~s}$, the robot rises to its maximum height of approximately $400 \mathrm{~mm}$, the robot completes the task of jumping $100 \mathrm{~mm}$ in height. From $0.8 \mathrm{~s}$ to 
$1.2 \mathrm{~s}$, the femur-patella joint angle changes by approximately $1.5 \mathrm{rad}$, the tibia-metatarsus joint angle changes by approximately $1.2 \mathrm{rad}$ and the metatarsus-tarsus joint angle changes by approximately $2.0 \mathrm{rad}$.

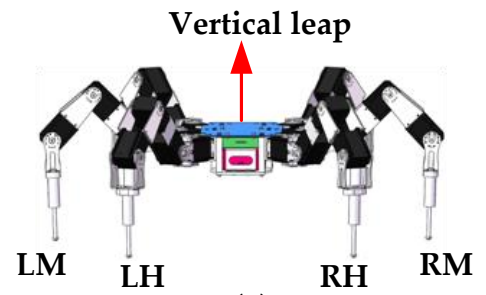

(a)

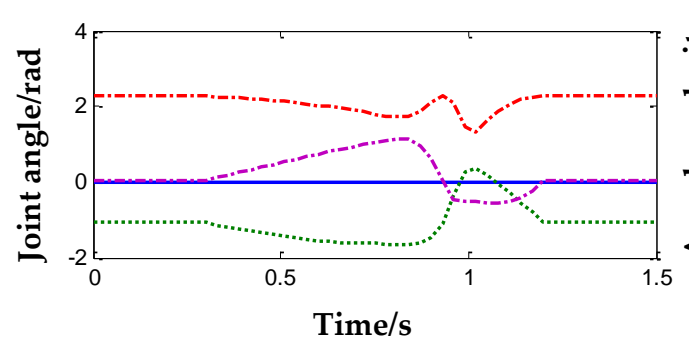

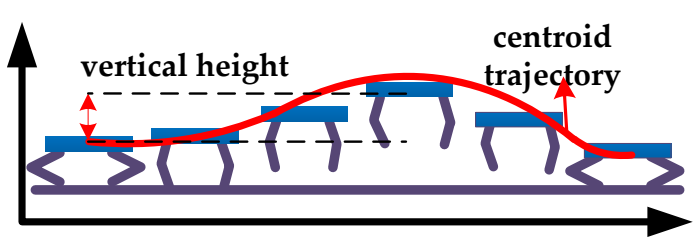

(b)

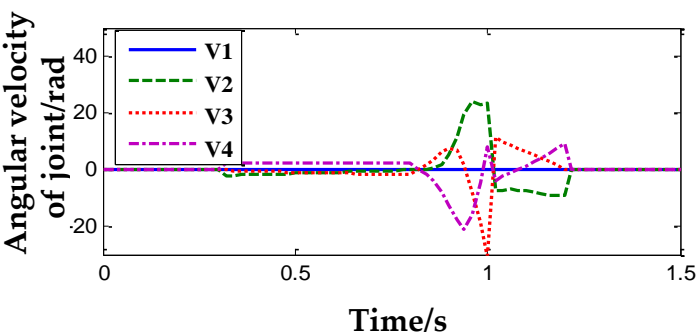

Time/s

tibia-metatarsus _.... metatarsus-tarsus joint joint

(c)

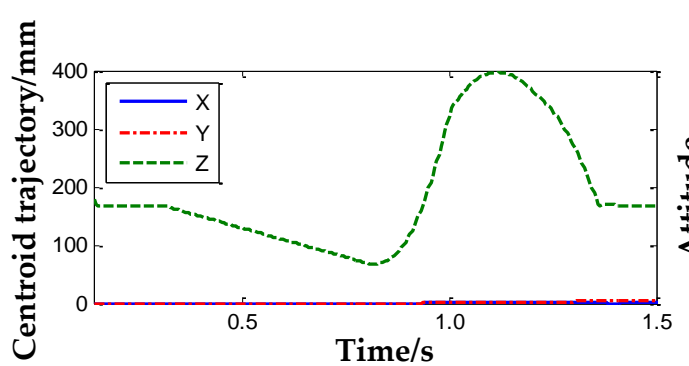

(d)

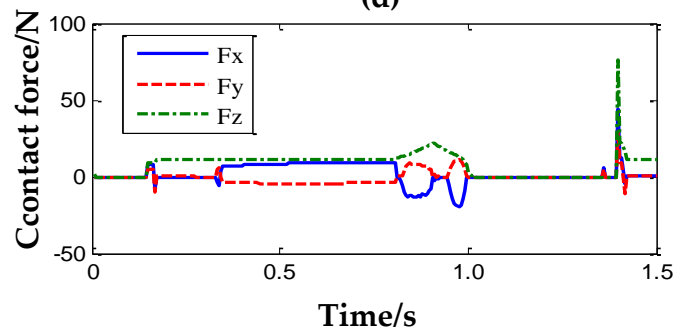

(f)

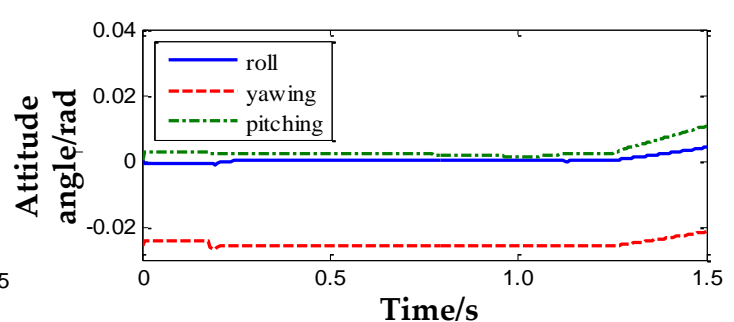

(e)

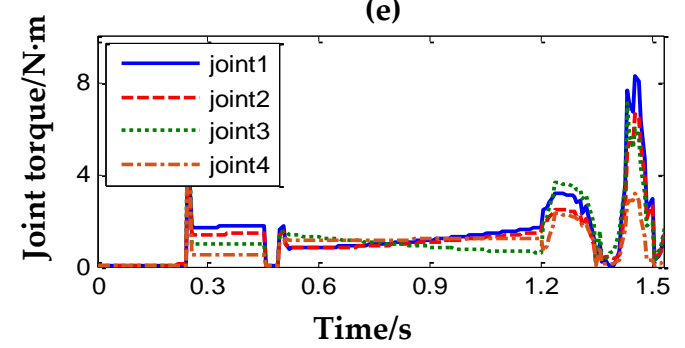

(g)

Figure 8. (a) Simulation platform of the virtual jump; (b) sketch of the robot vertically jumping; (c) joint angle curves of the robot leg; (d) trajectory of center of gravity (CoG); (e) attitude angle of robot; (f) contact force; and (g) joint torque.

As mentioned, the effective length of the robot's legs always changes during the vertical jump. For the robot to achieve stability, the change of the attitude angle is analyzed. The initial attitude angle of the robot is not $0 \mathrm{rad}$, which is due to the initial pose of the robot in Figure 8e. The roll angle holds at near $0 \mathrm{rad}$ when the robot jumps. The pitch angle holds at $0.002 \mathrm{rad}$ at approximately $0-1 \mathrm{~s}$, and the roll angle increases gradually to $0.01 \mathrm{rad}$ when the robot lifts off the ground. In the process of jumping, the yaw angle of the robot always holds at approximately $-0.025 \mathrm{rad}$. According to the simulation results, the robot has good stability in the vertical jump process. Figure $8 \mathrm{f}$ is the contact force of the foot tip for the robot in the vertical jump process. When the robot jumps vertically, it completes the 
upward jumping task through the contact force in the $\mathrm{Z}$ direction. The forces all cancel each other out which acts on the entire robot in the $X$ and $Y$ directions. As shown in Figure 8f, the initial contact force is $0 \mathrm{~N}$, which is due to the initial state of the robot. At $0.3-0.8 \mathrm{~s}$, the CoG slowly drops and the contact force remains constant; the contact force of $X$ is approximately $10 \mathrm{~N}$, the contact force of $\mathrm{Y}$ is 0 . The contact force $\mathrm{F}_{Z}$ of $\mathrm{Z}$ is approximately $12 \mathrm{~N}$, which is $1 / 6$ of the gravity of the robot. At $0.3-0.8 \mathrm{~s}$, the contact force of the foot tip increased rapidly, and the $Z$ directional force increased to $24 \mathrm{~N}$ at $1.0 \mathrm{~s}$, and decreased to $12 \mathrm{~N}$ after $1.0 \mathrm{~s}$. Overall, the vertical acceleration of the robot does not reach $0 \mathrm{~m} / \mathrm{s}^{2}$, the speed increases and reaches its maximum at $1 \mathrm{~s}$. After the robot jumps off the ground, the contact force is $0 \mathrm{~N}$ until the robot lands. Figure $8 \mathrm{~g}$ is the joint torque of the robot in the vertical jump process. At the initial stage of simulation, the robot foot does not touch the ground and the joint torque of the robot is $0 \mathrm{Nm}$. When the robot touches the ground, the joint torques changes slowly between $1 \mathrm{Nm}$ and $3 \mathrm{Nm}$ until the robot begins to adjust its posture at $0.5 \mathrm{~s}$. At $0.8-1.2 \mathrm{~s}$, the joint torque of the robot begins to increase. At $1.4 \mathrm{~s}$, the joint torque of the robot fluctuates wildly, and the maximum valve is approximately $8 \mathrm{Nm}$, which is caused by the impact of the robot landing.

According to these curves, we can see that the experimental data are consistent with what we expect from our projections; the robot could complete the task of jumping $100 \mathrm{~mm}$ in height while maintaining good stability.

\subsection{Sideways Jump}

The simulation experiment of the robot jumping sideways is carried out to verify whether the joint angle, attitude angle, jumping height and jumping distance are consistent with motion planning or not. Unlike the vertical jump, there is much variability in the joint kinematics and attitude with the sideways jump. At the beginning of the sideways jump, the lateral body axis began to rotate sideways in the jumping direction. The initial attitude is adjusted to prepare to jump. At the same time, the CoG drops and begins to store energy. During the acceleration phase of the sideways jump, the effective leg length of the robot extends quickly and the foot impacts the ground quickly, then the robot starts to take off. Figure $9 \mathrm{~b}$ shows a sketch of the robot jumping sideways. The initial height of the robot is $180 \mathrm{~mm}$, the jumping height is $100 \mathrm{~mm}$, and the jumping distance is $250 \mathrm{~mm}$.

During the robot's sideways jump, the legs in contact with the ground are first flexed and then extended. Each joint of the six legs has a different rotation angle. Before the acceleration phase begins, the CoG begins to drop and store energy at $0.3 \mathrm{~s}$ (Figure $9 \mathrm{~d}$ ); the legs on one side of the robot's body are obviously flexed, but those on the other side remain relatively small in the jumping direction. From $0.3 \mathrm{~s}$ to $0.8 \mathrm{~s}$, the robot body slows down and adjusts the attitude. At this instant, the femur-patella joint angle, tibia-metatarsus joint angle and metatarsus-tarsus joint angle begin to slowly change, and the coxal joint angle remain constant until the jump ends (Figure 9c). Then, the foot impacts the ground quickly the robot's legs start to extend and the robot begins to accelerate at approximately $0.8 \mathrm{~s}$. The joint angle begins to change quickly (Figure 9c), while the coxal joint angle of the middle leg remains unchanged; this is due to the robot's structure and path planning of the sideways jump. The displacement of the robot in the jumping direction begins to change, depending on the time at which the robot is in the air. Then, the robot leaves the ground and takes off, with the height of the CoG at $320 \mathrm{~mm}$ in this moment. The robot rises to its maximum height of approximately $420 \mathrm{~mm}$ and completes the task of jumping $100 \mathrm{~mm}$ (Figure 9d). At $1.4 \mathrm{~s}$, the robot will touch down. The robot is in the air for approximately $0.4 \mathrm{~s}$, while the jumping distance is $250 \mathrm{~mm}$ (Figure 9d). The robot jumps to the right. From $0.8 \mathrm{~s}$ to $1.2 \mathrm{~s}$, the femur-patella joint angle changes by approximately 2 rad, the tibia-metatarsus joint angle changes by approximately $2.5 \mathrm{rad}$ and the metatarsus-tarsus joint angle changes by approximately $0.5 \mathrm{rad}$ in the three left legs (Figure 9c). Concurrently, the femur-patella joint angle changes by approximately $1.5 \mathrm{rad}$, the tibia-metatarsus joint angle changes by approximately $2.5 \mathrm{rad}$ and the metatarsus-tarsus joint angle changes by approximately $1.0 \mathrm{rad}$ in the three right legs (Figure 9c). The attitude angle directly influences the stability of the robot when it jumps sideways in Figure 9c. The initial attitude angle of the robot depends on attitude planning. The roll angle 
always holds near 0.02 rad when the robot jumps, and the roll angle changes $0.1 \mathrm{rad}$ until the robot has landed. The pitch angle holds at near $0.2 \mathrm{rad}$. In the process of jumping, the yaw angle of the robot always holds at $0.02 \mathrm{rad}$; it increases gradually to $0.03 \mathrm{rad}$ when the robot has landed. According to the simulation results, the attitude angle of the robot is nearly invariable. Hence, the robot has good stability in the sideways jump process, which provides a good theoretical basis for the forward jump of the robot.

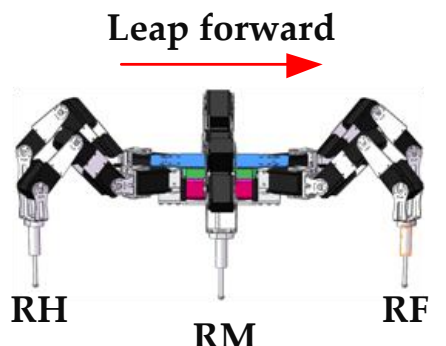

(a)
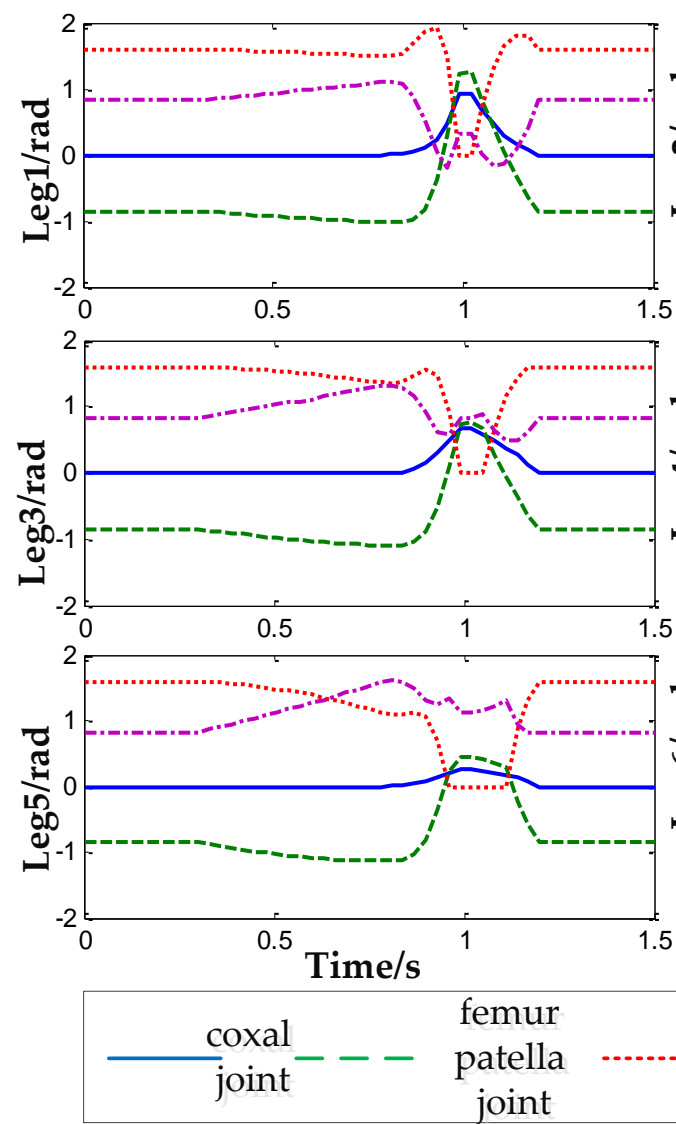

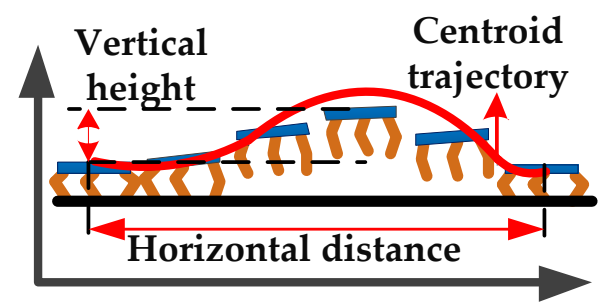

(b)
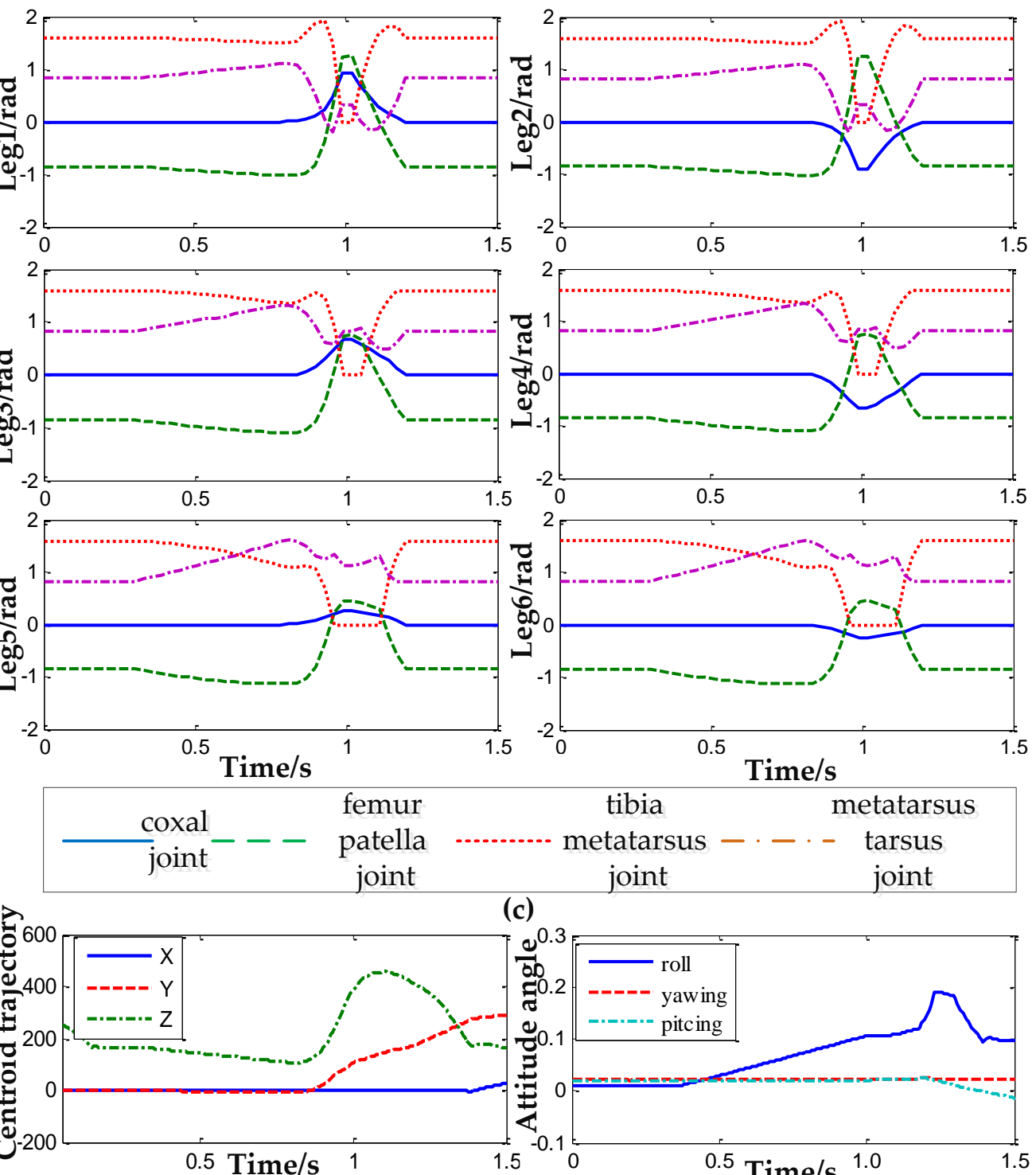

(d)

(e)

Figure 9. (a) Simulation platform of the sideways jump; (b) sketch of robot jumping sideways; (c) joint angle curves of the robot leg; (d) trajectory of the CoG; and (e) attitude angle of the robot. 
According to the simulation results, we can see that the experimental data are consistent with what we expect from our projections for the sideways jump. Therefore, the robot could complete the task of jumping $100 \mathrm{~mm}$ in height and $250 \mathrm{~mm}$ in distance, while maintaining good stability.

\subsection{Forward Jump}

The simulation of the robot jumping forward is carried out to verify whether the joint angle, attitude angle, jumping height and jumping distance is consistent with motion planning or not. The simulation results are seen in Figure 10. In the forward jump, the robot's body slowly moves backwards and downwards due to a slight flexion of the hind legs and the middle legs before the acceleration phase begins. The robot begins to store energy with the CoG dropping. At the same time, the initial attitude is adjusted in preparation to jump. Then, the robot starts to accelerate by extending its legs; the effective leg length increases quickly. Eventually, the robot takes off. Figure 10b shows a sketch of the robot jumping forward. The initial height of the CoG of the robot is $180 \mathrm{~mm}$, the jumping height is $100 \mathrm{~mm}$, and the jumping distance is $250 \mathrm{~mm}$.

While the robot jumps forward, the legs that have contact with the ground are first flexed and then extended. Each joint of the six legs has a different rotation angle (Figure 10c). Before the acceleration phase of the forward jump, the body rotates backwards as the hind legs and the middle of the robot body slightly flex in the jumping direction. Meanwhile, the robot adjusts its attitude by moving backwards and downwards. Then, the foot impacts the ground quickly, and the robot's legs start to extend and, at $0.8 \mathrm{~s}$, the robot begins to accelerate. At the same instant, the joint angle begins to quickly change (Figure 10c). At $1.0 \mathrm{~s}$, the robot loses contact with the ground and takes off; due to the accumulation of velocity, the rotation of the body is reversed. At this instant, the height of the CoG is $320 \mathrm{~mm}$. The robot rises to its maximum height of approximately $420 \mathrm{~mm}$ at $1.2 \mathrm{~s}$; it then completes the task of jumping $100 \mathrm{~mm}$ in height (Figure 10d). After this instant, none of the joint angles change (Figure 10c).

During the robot's forward jump, and the Coxal joint angles change by approximately 0.8 rad. The femur-patella joint angle changes by approximately $2 \mathrm{rad}$, the tibia-metatarsus joint angle changes by approximately $1.5 \mathrm{rad}$ and the metatarsus-tarsus joint angle changes by approximately $1 \mathrm{rad}$. At $1.4 \mathrm{~s}$, the robot lands on the ground. The robot is in the air for approximately $0.4 \mathrm{~s}$, and the jumping distance is $250 \mathrm{~mm}$ (Figure 10d). In Figure 10e, the initial attitude angle is not 0 , and the initial roll angle is $0.01 \mathrm{rad}$; the initial yaw angle is $0.025 \mathrm{rad}$; and the initial pitch angle is $0.02 \mathrm{rad}$, due to the initial pose of the robot. The roll angle changes by $0.02 \mathrm{rad}$ in the jumping process, and the roll angle decreased by $0.1 \mathrm{rad}$ when the robot landed. The pitch angle holds at $0.01 \mathrm{rad}$ in the jumping process, and it generated a fluctuation of $0.1 \mathrm{rad}$ when the robot landed. The yaw angle was almost constant until the robot landed, maintaining $0.015 \mathrm{rad}$. According to the simulation results, the robot has good stability in the forward jump process, which provides a good theoretical basis for the forward jump of the robot.

Observing the simulation result, we find that the experimental data are consistent with our projections of the forward jump. The robot could complete the task of jumping $100 \mathrm{~mm}$ in height and $250 \mathrm{~mm}$ in distance; it maintains good stability in the flying phase. 


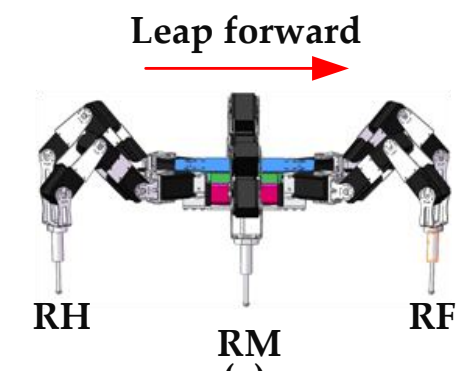

(a)

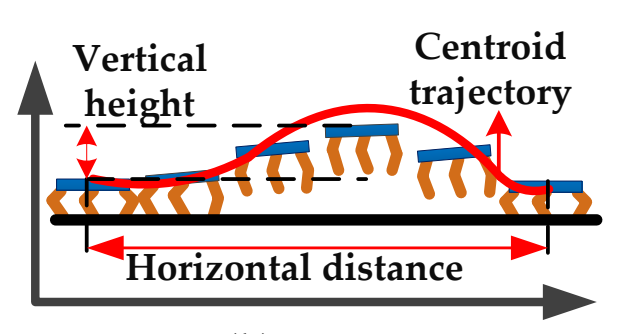

(b)
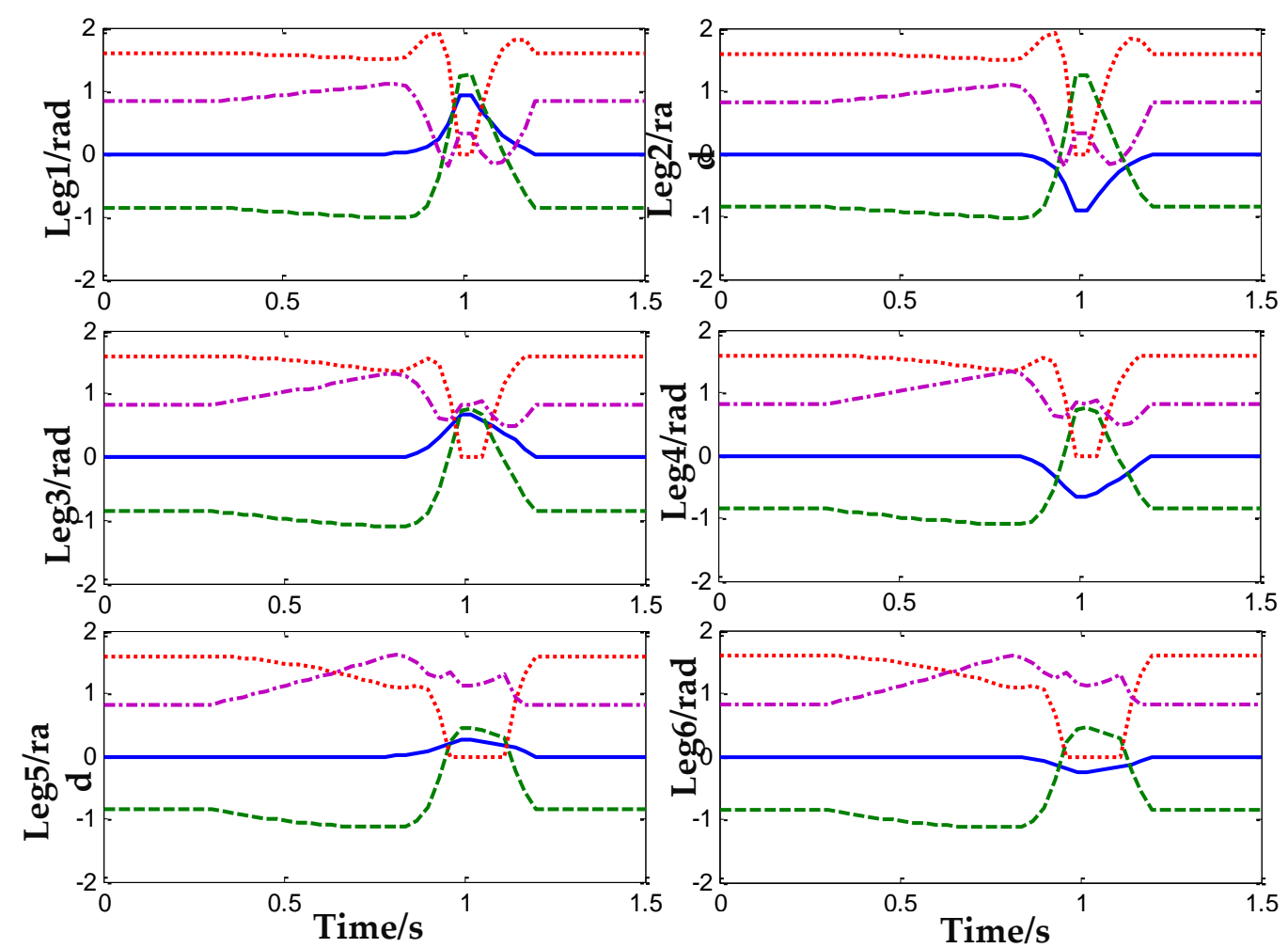

$\begin{array}{cccc}\text { coxal } & \text { femur patella } & \text { tibia metatarsus } & \text { metatarsus tarsus } \\ \text { joint } & \text { joint } & \text { joint } & \text { joint }\end{array}$

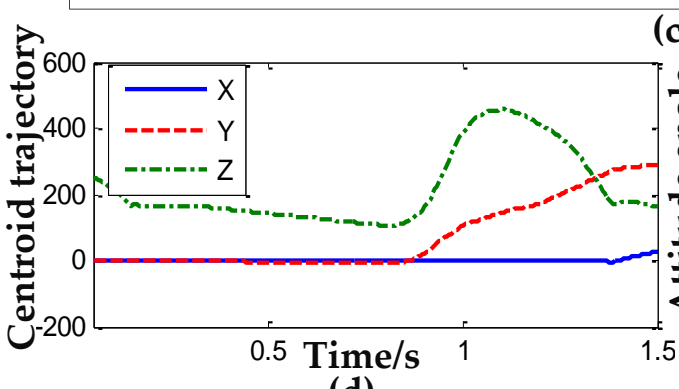

(d)

(c)

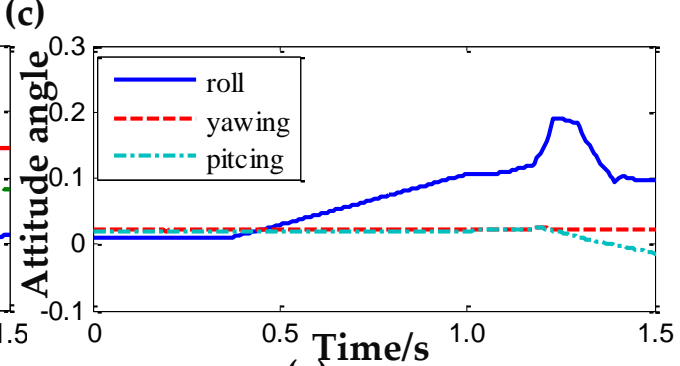

(e)

Figure 10. (a) Simulation platform of the forward jump; (b) sketch of the robot forward jumping; (c) joint angle curves of the robot leg; (d) trajectory of the CoG; and (e) attitude angle of robot.

\section{Discussion}

In this paper, research on the omnidirectional jump control of the hexapod robot based on the behavior mechanisms of jumping spiders was undertaken. In the first section, the jumping forms of several typical legged robots $[16,25,28,34]$ were described in detail. Normally, the more jumping force a robot has, the more difficult it is to control its jumping movement. However, the robot with more 
jumping force has a better capacity for avoiding obstacles. In the past decade, research on jumping robots has mainly focused on single-direction jumps, such as the vertical jump and the forward jump. However, this type of jumping robot must first adjust its posture and jumping direction when trying to avoid an obstacle. Therefore, the efficiency of the robot is greatly reduced. We study the change of the effective leg length and joint angle of the jumping spider, before proposing an omnidirectional jump control of the hexapod robot based on the behavior mechanisms of jumping spiders. Through a series of simulation experiments, we verify the possibility of an omnidirectional jump by comparing the simulation and experimental data with the data expected from our projections. Finally, the robot realizes rapid jumping in all directions under any circumstances.

There are several forms of DoF for robot legs, including 1DoF, 3DoFs and 4DoFs. Large DoF will greatly increase the complexity of motion controls, but smaller ones cannot satisfy the amount of motion required. The $1 \mathrm{DoF}$ model of a jumping robot leg is usually made by a hydraulic component [37] or an elastic component [38]. However, it is difficult to guarantee the stability of motion with such robots. Currently, 3DoFs for jumping robot legs is the main form, but does not have enough flexibility when the robot jumps omnidirectionally. In this paper, the jumping robot has adapted 4 DoFs-mechanical legs, which increases the flexibility of the robot when it jumps omnidirectionally. The attitude angle of the foot tip is obtained based on bionic kinematics, and the constraint of the foot tip's attitude angle is added to calculate the inverse kinematic. Since most studies are in a unidirectional jumping stage to the jumping robot, this type of robot cannot realize omnidirectional jumping, especially avoid obstacles quickly. In this paper, omnidirectional jumping has been proposed as it allows the robot to avoid and cross obstacles in all directions. We proposed a study based on bionic kinematics with redundant freedom, which could potentially solve the inverse kinematics for a bionic structure with redundant freedom and achieve to jump in many different types to robot. Furthermore, we proposed locomotion planning, especially attitude planning, by observing the behavior of the jumping spider.

\section{Conclusions}

In this paper, the difficulty of omnidirectional jumping for a jumping bio-mimetic spider robot was addressed. The theoretical contribution and novelty of this paper can be summarized as follows:

(1) The path of the robot, the initial attitude and the trajectory of the foot tip must be planned to complete the jumping task. In particular, the reasonable initial attitude angle of the robot can affect jumping height and distance.

(2) To satisfy the diversity of motion forms in robot jumping, each leg has 4DoFs. However, the 4 DoFs-mechanical leg is a redundant structure and we must find a constraint condition. According to the change curve of each joint angle in the process of spider jumping, we can obtain the attitude angle curve as the added constraint condition.

(3) Three kinds of jumps are verified on the jumping robot prototype: vertical jumps, sideways jumps and forward jumps. The proposed method is verified by a series of simulation experiments. The results indicate that the jumping robot could maintain stability and complete the task of jumping we planned, and the proposed spider-inspired jumping strategy could easily achieve an omnidirectional jump, and robot able to avoid obstacles quickly.

The results indicate that the robot can perform the omnidirectional jump according to the path planning of the CoG and the initial jumping attitude. The robot also has better stability, as observed by the attitude angle of the jumping robot during its jumps. Therefore, the robot can jump in any direction by providing the trajectory of the CoG and the initial attitude.

Acknowledgments: This study is supported by the National Natural Science Foundation of China (No. 51605039), the China Postdoctoral Science Foundation (No. 2016M592728), Shaanxi Postdoctoral Scientific Research Project (No. 2016BSHYDZZ26) and Open Foundation of the State Key Laboratory of Fluid Power Transmission and Control (No. GZKF-201610). 
Author Contributions: Yaguang Zhu designed the algorithm. Yaguang Zhu, Long Chen, and Qiong Liu designed and carried out the Simulation. Yaguang Zhu, Long Chen Rui Qin, Bo Jin and analyzed the data and wrote the paper. Bo Jin gave many meaningful suggestions about the structure of the paper.

Conflicts of Interest: The authors declare no conflict of interest.

\section{References}

1. Du, H.; Gao, F. Fault tolerance properties and motion planning of a six-legged robot with multiple faults. Robotica 2017, 35, 1397-1414. [CrossRef]

2. Lee, C.H.; Kim, S.H.; Kang, S.C. Double-track mobile robot for hazardous environment applications. Adv. Robot. 2003, 17, 447-459. [CrossRef]

3. Gillis, G.B.; Biewener, A.A. Hind limb Extensor Muscle Function during Jumping and Swimming in the Toad. J. Exp. Biol. 2000, 203, 3547-3563. [PubMed]

4. Wei, D.W.; Ge, W.J. Research Status and Development Trend of Jumping Robots. Robot 2014, 36, 502-512. [CrossRef]

5. Xu, H.Y.; Fu, Y.L.; Wang, S.G.; Liu, J.G. Research on biomimetic robotics. Robot 2004, 26, $283-288$.

6. Zak, M.; Rozman, J.; Zboril, F.V. Overview of Bio-Inspired Control Mechanisms for Hexapod Robot. Int. J. Comput. Inf. Syst. Ind. Manag. Appl. 2016, 8, 125-134.

7. Tom, W.; Michael, K.; Robert, J.F.; Reinhard, B. Jumping kinematics in the wandering spider Cupiennius salei. J. Comp. Physiol. A 2010, 196, 421-438. [CrossRef]

8. Dong, J.H.; Sangok, S.; Jongwoo, L.; Sangbae, K. High speed trot-running: Implementation of a hierarchical controller using proprioceptive impedance control on the MIT Cheetah. Int. J. Robot. Res. 2014, 33, 1417-1445. [CrossRef]

9. Zhu, X.Y.; Fan, J.Z.; Cai, H.G. Design of Control System for Frog-inspired Jumping Robot. Mach. Electron. 2011, 8, 63-67.

10. Umberto, S.; Cesare, S.; Paolo, D. Bioinspired Jumping Robot with Elastic Actuators and Passive Forelegs. Nat. Obs. 2009, 54, 329-338. [CrossRef]

11. Tom, W.; Michael, G.; Reinhard, B. Hydraulic leg extension is not necessarily the main drive in large spiders. J. Exp. Biol. 2011, 215, 578-583. [CrossRef]

12. Je, S.K.; Kyu, J.C. Development of an Insect Size Micro Jumping Robot. Living Mach. 2014, 8608, $405-407$. [CrossRef]

13. Kosa, G.; Ayali, A.; Hanan, U.B. Design of a Bio-Mimetic Jumping Robot. In Proceedings of the 27th Convention of Electrical and Electronics Engineers in Israel, Eilat, Israel, 14-17 November 2012; IEEE: Piscataway Township, NJ, USA, 2012.

14. Jindrich, D.L.; Full, R.J. Many-legged maneuverability: Dynamics of turning in hexapods. J. Exp. Biol. 1999, 202, 1603-1623. [PubMed]

15. Huang, X.G.; Hang, Z.Q. Kinematics analysis of frog-jumping robot. J. Mach. Des. 2012, 29, $23-25$.

16. Zhang, Q.; Chen, A.J. Study on the jumping kinematics of the bionic cricket robot. J. Mach. Des. 2010, 20, 38-41.

17. Zhang, X.F.; Qin, X.S.; Feng, H.S.; Tan, X.Q. Motion Analysis and Control of a Single Leg of Hydraulically Actuated Quadruped Robots during Vertical Jumping. Robot 2013, 35, 135-141. [CrossRef]

18. Lu, Q.Q.; Liu, C.H.; Liu, J.S.; Tang, K.Q. Simulation Analysis of Jumping Hexapod Robot Drived with Hydraulic. Mach. Des. Manuf. 2015, 8, 200-205.

19. Zhou, Y.; Cheng, L.; Chen, K. Energy supply strategy for one-legged jumping robot. J. Tsinghua Univ. 2015, 55, 273-278.

20. Li, Y.; Ge, W.J.; Fan, C.Q. Research and design on the foot of the jumping kangaroo robot based on adaptive structure. Mach. Des. Manuf. 2012, 5, 187-189.

21. Ge, W.J.; Shen, Y.W.; Yang, F. Research on the Driving Characteristics of Bionic Kangaroo jumping Robot. Mech. Eng. China 2006, 17, 856-861.

22. Zhang, W.T.; Ge, W.J.; Li, J.H.; Jiang, M.; Shen, P. Design and Research on the Power System of Kangaroo Jumping Robot. Robot 2008, 30, 359-363. 
23. Incaini, R.; Sestini, L.; Garabini, M.; Catalano, M.; Grioli, G.; Bicchi, A. Optimal Control and Design Guidelines for Soft Jumping Robots: Series Elastic Actuation and Parallel Elastic Actuation in Comparison. In Proceedings of the 2013 IEEE International Conference on Robotics and Automation (ICRA), Karlsruhe, Germany, 6-10 May 2013.

24. Tedeschi, F.; Carbone, G. Design Issues for Hexapod Walking Robots. Robotics 2014, 3, 181-206. [CrossRef]

25. Haynes, G.C.; Rizzi, A.A. Gaits and Gait Transitions for Legged Robots. In Proceedings of the 2006 IEEE International Conference on Robotics and Automation, Orlando, FL, USA, 15-19 May 2006.

26. Kikuchi, F.; Ota, Y.; Hirose, S. Basic Performance Experiments for Jumping Quadruped. In Proceedings of the 2003 IEEE International Conference on Intelligent Robots and Systems, Las Vegas, NV, USA, 27-31 October 2003.

27. Laksanacharoen, S.; Pollack, A.J.; Nelson, G.M.; Quinn, R.D.; Ritzmann, R.E. Biomechanics and Simulation of Cricket for Microrobot Design. In Proceedings of the 2000 IEEE International Conference on Robotics and Automation, San Francisco, CA, USA, 24-28 April 2000; IEEE: Piscataway Township, NJ, USA, 2002.

28. Sakakibara, Y.; Kan, K.; Hosoda, Y.; Hattori, M.; Fujie, M. Foot Trajectory for a Quadruped Walking Machine. In Proceedings of the IEEE International Workshop on Intelligent Robots and Systems, Ibaraki, Japan, 3-6 July 1990.

29. Birch, M.C.; Quinn, R.D.; Hahm, G.; Philips, S.M.; Drennan, B.; Fife, A.; Verma, H.; Beer, R.D. Design of a Cricket Microrobot. In Proceedings of the IEEE International Conference on Robotics and Automation, San Francisco, CA, USA, 24-28 April 2000; IEEE: Piscataway Township, NJ, USA, 2002.

30. Yang, Y.S.; Semini, C.; Guglielmino, E. Water vs. Oil Hydraulic Actuation for a Robot Leg. In Proceedings of the International Conference on Mechatronics and Automation, Changchun, China, 9-12 August 2009; IEEE: Piscataway Township, NJ, USA, 2009.

31. Thanhtam, H.; Sangyoon, L. Design of a Shape Memory Alloy-Actuated Biomimetic Mobile Robot with the Jumping Gait. Int. J. Control Autom. Syst. 2013, 11, 991-1000. [CrossRef]

32. Hyunsoo, S.; Sangyoon, L. Simulation and Experiments of a Four-Legged Robot That Can Locomote by Crawling and Jumping. In Proceedings of the 2014 IEEE International Conference on Robotics and Biomimetics, Bali, Indonesia, 5-10 December 2014.

33. Li, F.; Liu, W.; Fu, X.; Bonsignori, G.; Scarfogliero, U.; Stefanini, C.; Dario, P. Jumping like an insect: Design and dynamic optimization of a jumping mini robot based on bio-mimetic inspiration. Mechatronics 2012, 22, 167-176. [CrossRef]

34. Hoover, A.M.; Burden, S.; Fu, X.Y.; Shankar Sastry, S.; Fearing, R.S. Bio-Inspired Design and Dynamic Maneuverability of a Minimally Actuated Six-Legged Robot. In Proceedings of the IEEE RAS and EMBS International Conference on Biomedical Robotics and Biomechatronics, Tokyo, Japan, 26-29 September 2010; IEEE: Piscataway Township, NJ, USA, 11 November 2010.

35. Ackerman, J.; Seipel, J. Energetics of Bio-Inspired Legged Robot Locomotion with Elastically-Suspended Loads. In Proceedings of the IEEE International Conference on Intelligent Robots and Systems, San Francisco, CA, USA, 25-30 September 2011; IEEE: Piscataway Township, NJ, USA, 5 December 2011.

36. Scarfogliero, U.; Stefanini, C.; Dario, P. The use of compliant joints and elastic energy storage in bio-inspired legged robots. Mech. Mach. Theory 2009, 44, 580-590. [CrossRef]

37. Zhao, M.G.; Qiu, Y.; Chen, X. Control algorithm Based on Time Event for a Pneumatic Single-legged Jumping Robot. Robot 2012, 32, 525-530. [CrossRef]

38. Bjelonic, M.; Kottege, N.; Beckerle, P. Proprioceptive Control of an Over-Actuated Hexapod Robot in Unstructured Terrain. In Proceedings of the 2016 IEEE International Conference on Intelligent Robots and Systems, Daejeon, South Korea, 9-14 October 2016; IEEE: Piscataway Township, NJ, USA, 2016.

(C) 2018 by the authors. Licensee MDPI, Basel, Switzerland. This article is an open access article distributed under the terms and conditions of the Creative Commons Attribution (CC BY) license (http:/ / creativecommons.org/licenses/by/4.0/). 